\title{
Variable X-ray emission from the accretion shock in the classical T Tauri star V2129 Ophiuchi
}

\author{
C. Argiroffi ${ }^{1,2}$, E. Flaccomio ${ }^{2}$, J. Bouvier ${ }^{3}$, J.-F. Donati ${ }^{4}$, K. V. Getman ${ }^{5}$, S. G. Gregory ${ }^{6,7}$, G. A. J. Hussain ${ }^{8}$, \\ M. M. Jardine ${ }^{9}$, M. B. Skelly ${ }^{4}$, and F. M. Walter ${ }^{10}$ \\ ${ }^{1}$ DSFA, Univ. di Palermo, Piazza del Parlamento 1, 90134 Palermo, Italy \\ e-mail: argi@astropa.unipa.it \\ 2 INAF - Osservatorio Astronomico di Palermo, Piazza del Parlamento 1, 90134 Palermo, Italy \\ 3 UJF-Grenoble 1/CNRS-INSU, Institut de Planétologie et d'Astrophysique de Grenoble (IPAG) UMR 5274, 38041 Grenoble, \\ France \\ 4 LATT-UMR 5572, CNRS \& Univ. de Toulouse, 14 Av. E. Belin, 31400 Toulouse, France \\ 5 Department of Astronomy \& Astrophysics, 525 Davey Laboratory, Pennsylvania State University, University Park, PA 16802, \\ USA \\ 6 School of Physics, Univ. of Exeter, Stocker Road, Exeter EX4 4GL, UK \\ 7 California Institute of Technology, MC 249-17, Pasadena, CA 91125, USA \\ ${ }^{8}$ ESO, Karl-Schwarzschild-Strasse 2, 85748 Garching bei München, Germany \\ 9 School of Physics and Astronomy, Univ. of St Andrews, St Andrews, Scotland KY16 9SS, UK \\ 10 Department of Physics and Astronomy, Stony Brook University, Stony Brook, NY, 11794-3800, USA
}

Received 14 December 2010 / Accepted 28 February 2011

\section{ABSTRACT}

\begin{abstract}
Context. The soft X-ray emission from high density plasma observed in several CTTS is usually associated with the accretion process. However, it is still unclear whether this high density "cool" plasma is heated in the accretion shock, or if it is coronal plasma fed or modified by the accretion process.

Aims. We conducted a coordinated quasi-simultaneous optical and X-ray observing campaign of the CTTS V2129 Oph. In this paper, we analyze Chandra grating spectrometer data and attempt to correlate the observed X-ray emitting plasma components with the characteristics of the accretion process and the stellar magnetic field constrained by simultaneous optical observations.

Methods. We analyze a $200 \mathrm{ks}$ Chandra/HETGS observation, subdivided into two $100 \mathrm{ks}$ segments, of the CTTS V2129 Oph. For the two observing segments corresponding to two different phases within one stellar rotation, we measure the density of the cool plasma component and the emission measure distribution.

Results. The X-ray emitting plasma covers a wide range of temperatures: from 2 up to $34 \mathrm{MK}$. The cool plasma component of V2129 Oph $(T \approx 3-4 \mathrm{MK})$ varies between the two segments of the Chandra observation: during the first observing segment high density plasma $\left(\log N_{\mathrm{e}}=12.1_{-1.1}^{+0.6}\right)$ with high $E M$ at $\sim 3-4 \mathrm{MK}$ is present, whereas, during the second segment, this plasma component has lower $E M$ and lower density $\left(\log N_{\mathrm{e}}<11.5\right)$, although the statistical significance of these differences is marginal. Hotter plasma components, $T \geq 10 \mathrm{MK}$, vary on short timescales ( $\sim 10 \mathrm{ks})$, which are typical of coronal plasma. A clear flare, detected during the first segment, might be located in a large coronal loop (half length $>3 R_{\star}$ ).

Conclusions. Our observation provides additional confirmation that the dense cool plasma at a few MK in CTTS is material heated in the accretion shock. The variability of this cool plasma component on V2129 Oph may be explained in terms of X-rays emitted in the accretion shock and seen with different viewing angles at the two rotational phases probed by our observation. In particular, during the first time interval a direct view of the shock region is possible, while, during the second, the accretion funnel itself intersects the line of sight to the shock region, preventing us from observing the accretion-driven X-rays.
\end{abstract}

Key words. circumstellar matter - stars: coronae - stars: individual: V2129 Oph - stars: pre-main sequence - X-rays: stars stars: variables: T Tauri, Herbig Ae/Be

\section{Introduction}

During the pre-main sequence phase, low mass stars evolve from the embedded protostellar stage to the main sequence. In the first few million years of this transformation, they undergo a key phase in which, while gravitationally contracting, they accrete material from a circumstellar disk (see e.g. Bertout 1989; Kenyon \& Hartmann 1995). During this phase, low mass stars are called classical T Tauri stars (CTTS). When the accretion process ends, stars, still in the pre-main sequence but lacking the strong line emission characteristic of the previous phase, are classified as weak-line T Tauri stars (WTTS).
The accretion process in CTTS has been associated with the presence of high density plasma at a temperature of a few MK radiating soft X-rays. Many fundamental aspects of this link remain debated. Understanding the accretion process and its high-energy radiation is a key step in the understanding of the physics of pre-main-sequence low-mass stars and their circumstellar environment, because accretion strongly affects several properties of the central star itself: accretion determines stellar rotational velocities (Bouvier et al. 1993), and affects coronal activity (Preibisch et al. 2005); the accretion history in the early stages of stellar evolution might modify the stellar radius, hence its luminosity, up to ages of a few Myr (Baraffe et al. 2009); and 
the accretion process, because of the intense ionizing/heating radiation produced (UV and X-rays), influences the physics and the chemistry of circumstellar disks where planets are forming and may even determine their lifetime (e.g. Alexander et al. 2004; Ercolano et al. 2009).

The accretion process is a complex phenomenon in which a fundamental role is played by the stellar magnetic field: it truncates the inner part of the circumstellar disk and guides infalling material along its flux tubes toward the central star (Königl 1991). The magnetic field, interacting with the inner disk and channeling both inflowing (i.e. accreting) and outflowing material, mediates the angular momentum exchange between the star and the circumstellar material (Shu et al. 1994).

The accreting material from the inner disk approaches the star in (almost) free fall. The impact with the dense stellar atmosphere produces shocks at the footpoints of the accretion streams. Most of the kinetic energy of the infalling material is thus converted into heat, generating a region of higher temperature than the surrounding photosphere, a so-called hot spot. Evidence of these hot spots at $T \sim 10000 \mathrm{~K}$ is provided by optical/UV photometry and spectroscopy of CTTS.

From a theoretical point of view, accretion onto CTTS can produce significant amounts of X-rays, since the accreting material should impact onto the stellar surface with a high infall velocity $\left(v \sim 300-500 \mathrm{~km} \mathrm{~s}^{-1}\right)$, thus being heated in the accretion shocks up to temperatures of $\sim 2-6 \mathrm{MK}$. This hot material would radiate mostly in the soft $\mathrm{X}$-ray band $(0.1-1.0 \mathrm{keV}$, Gullbring 1994). The predicted X-ray luminosities in this band are high even for moderate mass-accretion rates (i.e. $L_{\mathrm{X}} \sim 10^{30} \mathrm{erg} \mathrm{s}^{-1}$ for $\dot{M} \sim 10^{-10} M_{\odot} \mathrm{yr}^{-1}$, Sacco et al. 2008). The observed $L_{\mathrm{X}}$ from CTTS in the $0.1-1.0 \mathrm{keV}$ band are, however, significantly lower than these predictions.

Observationally, CTTS are X-ray bright when one considers a broader $0.1-10 \mathrm{keV}$ band because of their very active coronae. Their intense magnetic fields, other than controlling accretion, are also responsible for the heating and confinement of large amounts of coronal plasma. Most of the X-ray emission from CTTS is actually due to very hot plasmas ( $T \geq 10 \mathrm{MK}$, much higher than that produced in accretion shocks) and is characterized by frequent flaring activity, clear evidence of coronal emission. Measurements of the density of the "cool" plasma $(T \sim 3-4 \mathrm{MK})$ in CTTS are, however, at odds with a coronal origin of at least part of the X-ray emission: in six out of the seven CTTS for which plasma densities have been measured (Kastner et al. 2002; Stelzer \& Schmitt 2004; Schmitt et al. 2005; Günther et al. 2006; Argiroffi et al. 2007; Güdel et al. 2007; Robrade \& Schmitt 2007; Huenemoerder et al. 2007) the cool plasma is significantly denser $\left(N_{\mathrm{e}} \geq 10^{11} \mathrm{~cm}^{-3}\right)$, than ever observed in the same-temperature plasma of older late-type stars (Testa et al. 2004) or in disk-bearing young stars without evidence of accretion (Kastner et al. 2004; Günther et al. 2010). This high density plasma cannot be interpreted in terms of steady coronal loops (Argiroffi et al. 2009). These findings indicate that the high density plasma component in CTTS is associated with the accretion process. In particular, it is suggested that this plasma is material heated in the accretion shock.

In addition to these studies of plasma densities, Güdel \& Telleschi (2007) found, while investigating the soft $(\sim 0.5 \mathrm{keV})$ X-ray flux level of CTTS and WTTS, that CTTS have higher soft $\mathrm{X}$-ray fluxes than WTTS. They suggest that this soft excess is due to shock-heated plasma, or to magnetically confined plasma interacting with (and/or originating from) accretion funnels.

Flaccomio et al. (2010), by observing the NGC 2264 star forming region, found that the time variabilities of the soft X-ray and optical luminosities are correlated in CTTS (but not correlated in WTTS). This correlation may imply that X-rays and optical radiation are both produced in the accretion shock and modulated by a time-variable accretion rate and/or by the stellar rotation. Supporting an idea previously proposed by Gregory et al. (2007), Flaccomio et al., however, favored a different scenario in which both the optical and soft X-ray variability are produced by a variable absorption, likely due to disk warps, as proposed by Bouvier et al. (2007) for the CTTS AA Tau.

All these results clearly indicate that the soft X-ray emission from high density plasma is somehow linked to the accretion process. Different scenarios have been developed to explain this link, but many fundamental points remain unclear. The final evidence, defining whether the high density cool plasma component is material heated in the accretion shock (Kastner et al. 2002), or coronal plasma somehow modified/affected by the accretion process (Güdel et al. 2007; Brickhouse et al. 2010), is still lacking. A fundamental role may also be played by a variable or non-uniform absorption produced by accretion streams and inner disk warps in CTTS.

A few high quality X-ray datasets of CTTS have provided tighter observational constraints that partially disagree with the predictions of current accretion-shock models. The high-density cool-plasma components of TW Hya cannot be explained by the shock of a single accretion stream (Brickhouse et al. 2010), but models with multiple streams are required (Sacco et al. 2010). Moreover, the shock-heated plasma model, applied to the observed X-rays from CTTS, provides significantly underestimated accretion rates with respect to measurements based on optical indicators (a factor of 10 or even more, Argiroffi et al. 2009; Curran et al. 2011). Local absorption of X-rays by either the surrounding stellar atmosphere (Drake 2005) or the pre-shock infalling material could play a fundamental role in determining the amount of X-ray emission escaping from the shock region (Sacco et al. 2010).

Determining the X-ray luminosity that emerges from the shock region, other than when interpreting the observations, is fundamental to: i) constraining the amount of high-energy radiation that heats and ionizes the circumstellar environment; and, ii) delineating the energy balance in the shock region.

To address the remaining open questions and improve our understanding of the accretion phenomenon, we have planned and performed a campaign of simultaneous multiwavelength observation of a CTTS. The main objective was to simultaneously constrain and correlate the characteristics of the accretion process, the stellar magnetic field, and the X-ray emitting plasma, including within both the coronal and the accretion-related components (Gregory et al. 2009).

For this study, we selected V2129 Oph, a CTTS in the $\rho$ Ophiuchi star-forming cloud and already one of the targets of the Magnetic Protostars and Planets (MaPP) project (Donati et al. 2009), whose main goal is to study the large-scale magnetic fields of CTTS using time-series of polarized Zeeman signatures.

In our study of V2129 Oph, we use several datasets, that were acquired simultaneously or quasi-simultaneously. V2129 Oph was monitored by optical spectroscopy and photometry from early May 2009 to mid July 2009. Its observational dataset includes: high resolution X-ray spectroscopy obtained with the Chandra High Energy Transmission Grating Spectrometer (HETGS), optical spectroscopy at both low and high spectral resolution (SMARTS/RC Spectrograph, SMARTS/Echelle Spectrograph, ESO/HARPS), 
optical polarized spectroscopy (ESPaDOnS), and NIR and optical photometry (SMARTS/Andicam time series).

Results based on the SMARTS and ESPaDOnS data, including Zeeman-Doppler brightness and magnetic fields maps, are presented by Donati et al. (2011) in a companion paper. In this paper, we present our analysis of the X-ray Chandra/HETGS observation, taking advantage, in the interpretation, of available results obtained from the optical data. Magnetic field extrapolation, based on the Zeeman-Doppler maps, will be described in forthcoming papers and will allow us to provide a threedimensional description of the open (wind-bearing) and closed (X-ray bright) field lines, and of the magnetic field structures through which accretion occurs (Jardine et al. 2008), enabling a more detailed comparison with the present X-ray observations.

We report the main properties of V2129 Oph in Sect. 2. Section 3 contains the analysis of the X-ray data, including short timescale variability (Sect. 3.3), and long timescale variability in which the analysis of high resolution X-ray spectra is possible (Sect. 3.4). Our main results are summarized in Sect. 4 and discussed in Sect. 5.

\section{Properties of V2129 Oph}

V2129 Oph is a CTTS belonging to the $\rho$ Oph star-forming region at a distance of 120 pc. It is a K5 star, with $M=1.35 M_{\odot}$, $R_{\star}=2.1 R_{\odot}$ (see Donati et al. 2011, for details). It has a lower mass companion, separated by $0.6^{\prime \prime}$. This companion displays a $K$-band flux ratio of $\sim 0.09$ with respect to V2129 Oph (McCabe et al. 2006). Using the evolutionary models of Siess et al. (2000), the mass of the secondary is inferred to be $\sim 0.1 M_{\odot}$. Being located at a sky-projected distance of $\sim 70$ AU from V2129 Oph, we can reasonably assume that it does not significantly affect its magnetosphere and accretion process. V2129 Oph has strong and variable $\mathrm{H} \alpha$ emission $(E W \sim 10-14 \AA$, Bouvier \& Appenzeller 1992). We selected V2129 Oph for this campaign because it is bright in both the optical and the X-ray band, is affected by moderate interstellar absorption $\left(A_{\mathrm{V}} \approx 0.6\right.$, Donati et al. 2007), and because, thanks to its rotational period $\left(P_{\text {rot }}=6.53 \mathrm{~d}\right.$, Shevchenko \& Herbst 1998; Grankin et al. 2008) and inclination angle $(i \approx 60 \mathrm{deg}$, Donati et al. 2011), can be studied with techniques that exploit the rotational modulation of surface features. V2129 Oph is the first CTTS for which Zeeman-Doppler maps of the surface magnetic fields were obtained (Donati et al. 2007) from time series of spectropolarimetric observations performed in 2005. Following this study, Jardine et al. (2008) extrapolated the surface magnetic field maps and inferred the characteristics of the accretion funnels and the coronal plasma of V2129 Oph at the time of the 2005 campaign. No coordinated X-ray observations were performed at that time, nor prior to our observation, V2129 Oph having only been observed in X-rays by ROSAT with low sensitivity and spectral resolution.

During our 2009 observing campaign, we acquired a new ESPaDOnS time series during 1-14 July, almost simultaneously with the Chandra observation (27-29 June 2009, see Sect. 3). The derived properties of V2129 Oph at the time of the Chandra observation are presented by Donati et al. (2011). A hot spot covering $\sim 2.5 \%$ of the stellar surface and located at high latitude ( $60 \mathrm{deg}$ ) was detected in Zeeman-Doppler maps of the Ca II IRT excess emission. This excess emission is powered by the accretion process, hence indicates the location of the footpoint of the main accretion stream in the visible part of the stellar surface. Donati et al. find that the radial component of the magnetic field has its peak intensity $(\sim 4 \mathrm{kG})$ in this same high latitude region, thus supporting its identification as the base of the accretion funnel, i.e. the magnetic flux tube that channels the accreting material. This accretion funnel is likely trailing the hot spot (i.e. its base) during the stellar rotation, as indicated by the periodic red-shifted absorption observed in the profiles of the Balmer lines. The intensity of the magnetic field suggests that the inner disk is truncated at a distance of 7.2 $R_{\star}$. The accretion rate of V2129 Oph appears to be quite stable during the observing campaign (apparent variations being within a factor 2) with an average value of $\log \dot{M}=-9.2$, where $\dot{M}$ is in units of $M_{\odot} \mathrm{yr}^{-1}$.

The upper part of Fig. 1 shows surface maps of the excess emission and the radial component of the magnetic field from Donati et al. (2011). The maps are shown as viewed from the observer at rotational phases corresponding to the beginning and the end of each of the two Chandra exposures comprising our $\mathrm{X}$-ray observation.

\section{Chandra observation and data analysis}

V2129 Oph was observed by Chandra/HETGS on 27-29 June 2009, for a total exposure of $200.4 \mathrm{ks}$. The observation, performed during two consecutive orbits, was divided into two segments (obs. id. 9943 and 9944, hereafter seg. 1 and seg. 2), with durations of 98.9 and $101.7 \mathrm{ks}$, and separated by $88.6 \mathrm{ks}$. The rotational phases of the surface maps of excess Ca II emission and radial magnetic field (Donati et al. 2011), corresponding to the beginnings and ends of the Chandra exposures, are shown in the upper part of Fig. 1. Rotational phases referenced throughout this paper are referred to the ephemeris defined in Donati et al. (2007).

\subsection{Data preparation}

The HETGS onboard Chandra (Canizares et al. 2005) is composed of two gratings, the High Energy and the Medium Energy Gratings (HEG and MEG). The HEG and MEG first order spectra cover the $1-15$ and $2-30 \AA$ ranges, with resolutions of 0.012 and $0.023 \AA(F W H M)$, respectively.

We reprocessed the Chandra/HETGS data using CIAO 4.2 (CALDB version 4.3.1) starting from the level 1 event file and following the standard processing threads described in the observatory website. For the analysis, we used both the dispersed HEG and MEG photons, and the zeroth order image. For the zeroth order image, source photons were extracted from a circular region centered on the source position (with radius of 5 pixels or $\sim 2.5^{\prime \prime}$, expected to encircle $>95 \%$ of the source photons), while the background was taken from a concentric annulus (with 10 and 15 pixels as inner and outer radii). HEG and MEG dispersed spectra were extracted from the standard rectangular regions aligned with the dispersion directions.

\subsection{Zeroth order image and CCD-resolution spectrum}

We inspected the shape of the point spread function (PSF) of the zeroth order image to assess the possible contribution to the detected X-rays by the low-mass companion to V2129 Oph. With a $0.6^{\prime \prime}$ separation and the Chandra spatial resolution, the companion might be marginally resolved if X-ray bright. The PSF, however, did not provide evidence of asymmetry. We therefore assume that the observed X-rays are all from V2129 Oph. This agrees with the expected low X-ray luminosity of the secondary 


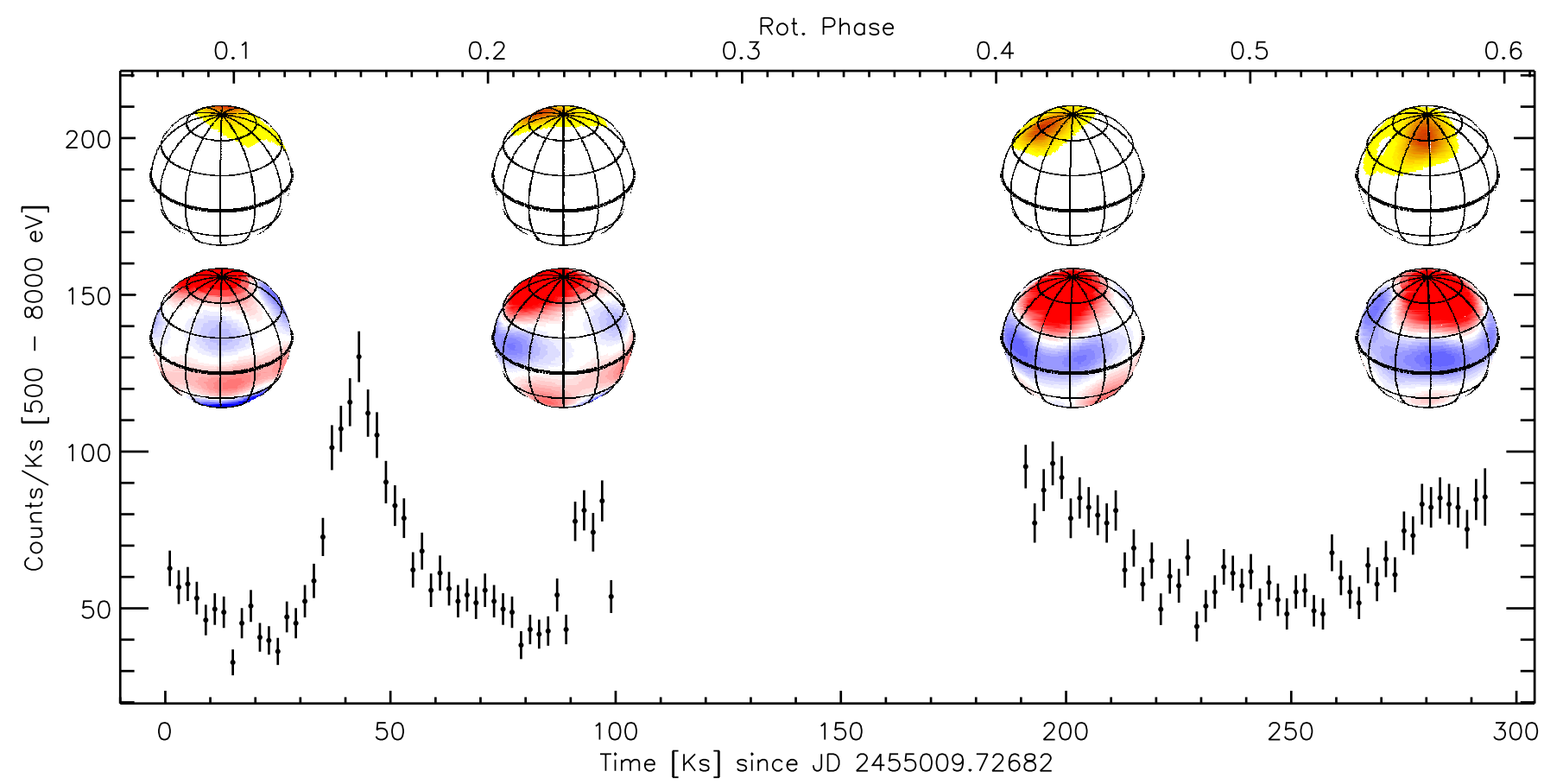

Fig. 1. Chandra light curve in the $0.5-10.0 \mathrm{keV}$ band combining events from the zeroth-order image and the HEG and MEG first-order spectra, with $2 \mathrm{ks}$ time bin. Shown above the light curve are images of the excess emission due to accretion (upper row) and of the radial component of the magnetic field (lower row) as reconstructed with quasi-simultaneous Zeeman-Doppler imaging by Donati et al. (2011). Colors scales are as in Donati et al. (red and blue in the lower row indicate positive and negative B-fields, respectively). The images refer to rotational phases at the beginning and the end of each of the two X-ray exposures.

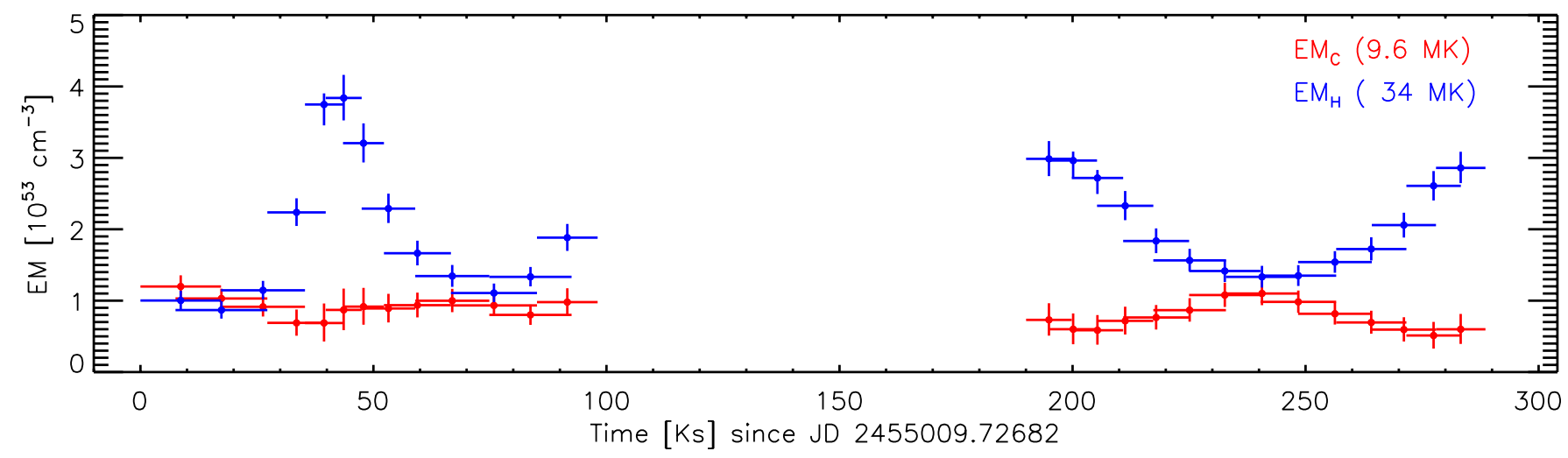

Fig. 2. Emission measure vs. time. Red and blue points refer to the $E M$ of the cool and hot components, respectively. See Sect. 3.3 .2 for details.

that, given the mass $-L_{X}$ relation of Preibisch et al. (2005), is $\sim 8 \times$ $10^{29} \mathrm{erg} \mathrm{s}^{-1}$, about $3 \%$ of the observed $L_{\mathrm{X}}$.

We then analyzed the CCD ACIS spectrum obtained from the undispersed zeroth order image with the main goal of constraining the amount of absorption affecting X-rays from V2129 Oph. Absorption, parametrized as usual by the equivalent hydrogen column density $N_{\mathrm{H}}$, alters the shape of the X-ray spectrum mostly at energy below $1 \mathrm{keV}$ (for modest values of $N_{\mathrm{H}}$ ) and, in this energy range, the zeroth order spectrum has a higher signal-to-noise ratio (hereafter $S / N$ ) than HEG and MEG spectra.

We considered the zeroth order spectrum integrated over the whole Chandra observing time (we found no evidence of $N_{\mathrm{H}}$ variations within the Chandra observation, see Sect. 3.3). The spectrum, binned between 0.5 and $10 \mathrm{keV}$ to ensure a $S / N$ of 10.0 per bin, was analyzed using XSPEC. A two-temperature thermal model (APEC, in XSPEC) subject to interstellar absorption (WABs) was found to adequately fit the observed spectrum. Elemental abundances were fixed to the reference values obtained by Maggio et al. (2007) from the X-ray emission of young stars of the Orion nebula cluster (ONC). We determined the statistical quality of the fits from the null probability implied by the $\chi^{2}$ and the number of degrees of freedom. The parameters of the emission model are $T_{1}=9.6_{-1.0}^{+1.5}, T_{2}=34_{-4}^{+5} \mathrm{MK}$, $\log E M_{1}=52.94 \pm 0.11$, and $\log E M_{2}=53.21_{-0.07}^{+0.05}$ (with $E M$ in units of $\left.\mathrm{cm}^{-3}\right)$. The best-fit model value for the hydrogen column density, $N_{\mathrm{H}}$, is $\left(8_{-4}^{+6}\right) \times 10^{20} \mathrm{~cm}^{-2}$, fully compatible with the value estimated from $A_{\mathrm{V}}$ using the standard conversion relation $N_{\mathrm{H}}=1.6 A_{\mathrm{V}} \times 10^{21}$ (Vuong et al. 2003). We finally note that the best-fit model value for $N_{\mathrm{H}}$ does not change significantly if the zeroth order spectrum is fit adopting the abundances that result from our analysis of the dispersed spectrum (Sect. 3.4.2). 


\subsection{Short-term variability}

To investigate time-variability on short timescales, we combined the zeroth order undispersed photons with the first order HEG and MEG spectra, collected during selected time-intervals. Combining these three datasets allowed us to increase the $S / N$, hence explore timescales as short as a few hours.

\subsubsection{X-ray light curve}

We first constructed a combined background-subtracted light curve from the arrival times of photons in source and background regions of both the zeroth order image and the HEG and MEG dispersed spectra. For the zeroth order image, source and background photons were extracted as indicated in Sect. 3.1. For the HEG and MEG spectra, to increase the $S / N$, we selected non-standard regions: first order source events were extracted within $2^{\prime \prime}$-wide strips along the direction of dispersion; and background events were extracted from strips, extending from 7.2 to $14.4^{\prime \prime}$ perpendicular to dispersion direction, on each side of each spectrum. Considering both observing segments, we collected, after background subtraction, a total of 5852, 2034.5, and 5341.25 photons (in the $E=0.5-10 \mathrm{keV}$ band) from the zeroth order image, the HEG spectrum, and the MEG spectrum, respectively.

Figure 1 shows the zeroth + first order light curve of our two observing segments, using $2 \mathrm{ks}$ binning. The time elapsed since the beginning of the observation and the rotational phase of V2129 Oph are given in the lower and upper $x$-axis, respectively.

The light curve shows a clear flare toward the middle of seg. 1, lasting $\sim 10 \mathrm{~h}$ and with a peak count-rate $\sim 3$ times higher than the pre-flare level, and what appears as a smaller flare toward the end of the same segment. During seg. 2, a seemingly more gradual variability is observed with a slow decay in the first $\sim 35 \mathrm{ks}$, and a similarly slow rise in the last $\sim 35 \mathrm{ks}$.

\subsubsection{Time-resolved spectroscopy}

A clearer insight into the physical origin of the observed variability might come from a time-resolved spectral characterization of the X-ray emission. To this end, we have extracted spectra in time intervals containing a total of 900 counts in the combined zeroth order and first order (HEG+MEG) light curve. We oversampled the light curve so that each $i$ th interval overlaps with the $(i \pm 1)$ th ones but is fully independent of the $(i \pm 2)$ th intervals. For each interval, three spectra were extracted: the zeroth order ACIS spectrum, and the two first order HEG and MEG spectra. These spectra were binned between 0.5 and $10 \mathrm{keV}$ so to have a $S / N$ of 3.0 per bin. Simultaneous spectral fits to the three spec$\operatorname{tra}^{1}$ in the $0.5-10 \mathrm{keV}$ band were then performed with XSPEC, using both the $\chi^{2}$-statistic and the Cash-statistic.

We adopted one and two temperature thermal models (APEC, in XSPEC), including the effect of interstellar absorption (WABS), with plasma abundances fixed to the values of Maggio et al. (2007). Fits were performed by both considering the absorption, i.e. the hydrogen column density $N_{\mathrm{H}}$, as a free parameter and fixing $N_{\mathrm{H}}$ to $8 \times 10^{20} \mathrm{~cm}^{-2}$, the value obtained in Sect. 3.2 for the time-averaged zeroth order spectrum. The statistical quality of

\footnotetext{
${ }^{1}$ Note that for the analysis of the time-averaged spectrum we used the zeroth order data only. This was justified by the higher $S / N$ of the zeroth order spectrum at low energies and the need to avoid cross-calibration issues. For the lower signal spectra discussed here, the statistical uncertainties are always larger than the systematic ones and cross-calibration issues are therefore less important.
}

the fits was evaluated by considering the null probability associated with $\chi^{2}$ and the number of degrees of freedom. Two thermal components were generally required to obtain acceptable fits, especially when fixing the $N_{\mathrm{H}}$. No evidence of absorption variability was found and we thus favored models with fixed $N_{\mathrm{H}}$. Although two temperatures were always required for an acceptable fit, no evidence of a significant evolution of the temperatures was found, the only exception being the time interval corresponding to the rise phase of the flare where a different modeling approach indicates a higher temperature for the hot component (see Sect. 3.3.3). Statistically acceptable fits could be obtained by fixing the temperatures to those obtained from the average spectrum (Sect. 3.2). This simply indicates that the emission measure distribution $(E M D)$ of the plasma, while varying significantly in time, can at all times be schematically represented by two dominant thermal components of fixed temperatures and that no significant additional component can be discerned with the limited resolution and signal of the spectra in individual time intervals ${ }^{2}$. Fixing the $N_{\mathrm{H}}$ and the two temperatures, the only free parameters left in the fits are the emission measures, $E M$, of the two components. Figure 2 shows the time evolution of the cool and hot $E M$. A hardening of the spectrum at times of high countrates is observed (and is also found by a simpler hardness-ratio analysis) both during the flares (see Sect. 3.3.3) in seg. 1 and in the more gradual evolution in seg. 2.

From the above analysis, the absorption-corrected X-ray luminosity of V2129 Oph ranges, during our Chandra observation, from 2.1 to $5.5 \times 10^{30} \mathrm{erg} \mathrm{s}^{-1}$ in the $0.5-10.0 \mathrm{keV}$ band.

\subsubsection{Flare analysis}

Flares offer the opportunity to constrain the spatial distribution of coronal plasma. In particular, the length of the magnetic loops where flares occur can be estimated from the evolution of observable plasma properties during both the rise and the decay phases (Reale et al. 1997; Reale 2007). We thus analyzed in detail the large flare detected during the first observing segment.

To apply the diagnostic based on the decay phase, the heating occurring during the decay must be characterized. This is done by studying the time evolution of the plasma temperature and density. In our case, however, a time-resolved spectral analysis yielded no evidence of a significant temperature evolution during the decay phase, preventing the application of this method.

An estimate of the loop length could instead be obtained from the rise phase of the flare (Reale 2007). The quantities used to derive the loop length are: the time $t_{0}$ at which the loop temperature reaches its maximum; the corresponding plasma temperature $T_{0}$; the time at which the plasma density is maximum, $t_{\mathrm{M}}$; and the corresponding plasma temperature, $T_{\mathrm{M}}$. Both $t_{0}$ and $t_{\mathrm{M}}$ are relative to the beginning of the flare and the temperatures refer to the apex of the loop. Two independent estimates of the loop half length $L$ are then given by Eqs. (12) and (13) of Reale (2007):

$$
L \approx 3 \Psi^{2} T_{0}^{1 / 2} t_{\mathrm{M}} \quad L \approx 2.5 \frac{\Psi^{2}}{\ln \Psi} T_{0}^{1 / 2}\left(t_{\mathrm{M}}-t_{0}\right)
$$

where $\Psi$ is $T_{0} / T_{\mathrm{M}}, L$ is in units of $10^{9} \mathrm{~cm}, T_{0}$ is in units of $10^{7} \mathrm{~K}$, and $t_{0}$ and $t_{\mathrm{M}}$ are in ks.

We applied these diagnostics to the flare of V2129 Oph by analyzing the zeroth + first order HEG and MEG events. The

2 We have also repeated the analysis with a coarser time-binning, i.e. adopting intervals that contain 2000 counts, instead of 900, with analogous results. 


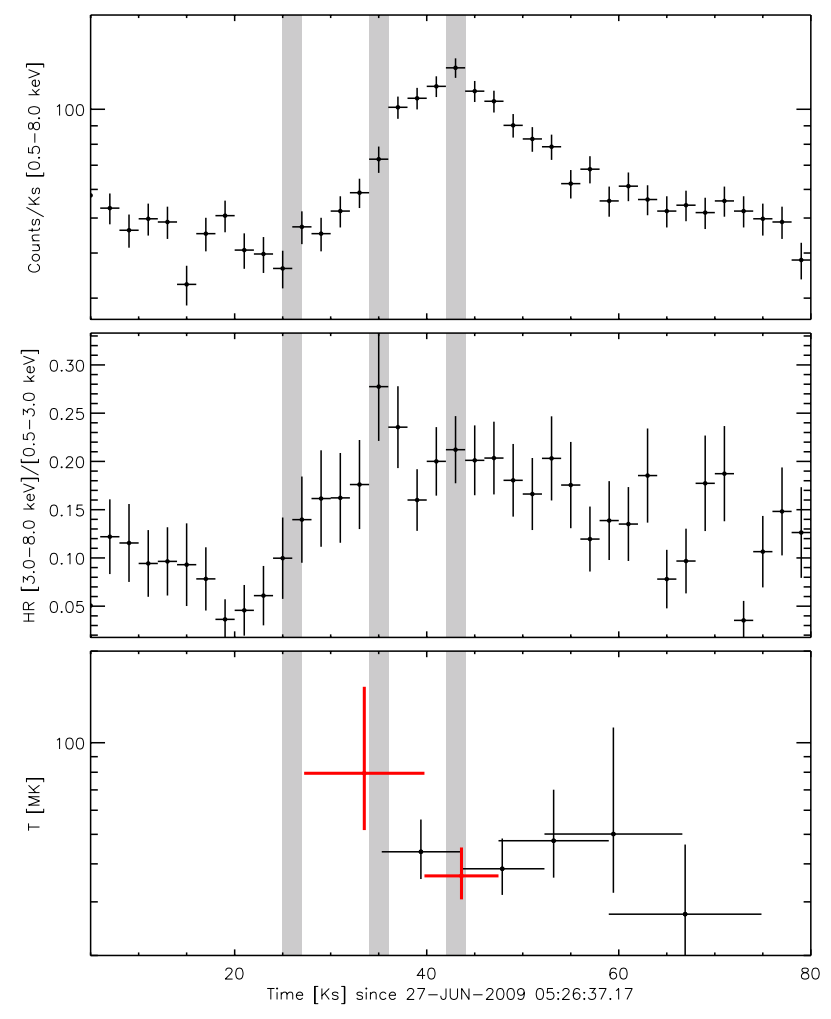

Fig. 3. Upper panel: Chandra X-ray light curve (the same shown in Fig. 1) of the flare. Middle panel: hardness ratio $(H R)$, defined as the ratio of events in the $3.0-8.0 \mathrm{keV}$ band to events in the $0.5-3.0 \mathrm{keV}$, versus time. Lower panel: temperature of the hot plasma component during flare evolution. Red points mark the maximum temperature and the temperature measured at the time in which the count rate is maximum.

times $t_{0}$ and $t_{\mathrm{M}}$ were estimated by inspecting the time evolution of the count-rate and hardness ratio ( $H R$, the ratio of the count rate in the hard to that in the soft band). The time of maximum density corresponds to the time of maximum count rate, this latter being approximately proportional to the $E M$ of the plasma in the flaring loop, thus assuming that the loop volume is constant, a proxy of the plasma density. The time of maximum temperature corresponds to the time of maximum $H R$, since the $H R$ increases for increasing temperature of the flaring plasma.

The upper panel of Fig. 3 shows the observed count rate in the $0.5-8.0 \mathrm{keV} \mathrm{X}$-ray band during the flare. We estimate that the flare starts $26 \pm 1 \mathrm{ks}$ after the beginning of the Chandra observation, and that the density peak occurs at $t_{\mathrm{M}}=43 \pm 1 \mathrm{ks}$. The middle panel of Fig. 3 shows the evolution of the $H R$, evaluated by adopting $0.5-3.0$ and $3.0-8.0 \mathrm{keV}$ as the soft and hard bands, respectively, to maximize the sensitivity to high temperatures. The maximum temperature occurs at $t_{0}=35 \pm 1 \mathrm{ks}$. The beginning of the flare and the times of maximum temperature and density are indicated in Fig. 3 with vertical gray bands.

We determined the temperatures $T_{0}$ and $T_{\mathrm{M}}$ by performing time-resolved spectral fittings. The adopted time intervals and the resulting extracted spectra are the same as defined in Sect. 3.3.2. Being interested in the temperatures of the flaring plasma, we must subtract the X-ray emission from the "quiescent" corona. We do so by assuming that the X-ray emission of the quiescent corona remains constant during the flare and is closely represented by the X-ray spectrum registered just before the beginning of the flare (in particular, with reference to Fig. 2, during the second interval centered at $t \sim 18 \mathrm{ks})$. We thus first derived a spectral model for the quiescent emission, then fitted the spectra of the "flaring" time-intervals assuming a spectral model composed of the sum of the quiescent spectrum (with fixed parameters) and an optically-thin isothermal plasma component (APEC, in XSPEC) subject to the same interstellar extinction as derived from the time-averaged spectral analysis $\left(N_{\mathrm{H}}=8 \times 10^{20} \mathrm{~cm}^{-2}\right)$. This latter component, describing the flaring plasma, is characterized by two fit-parameters, the temperature $T$ and the emission measure $E M$. The resulting best-fit temperatures are shown in the lower panel of Fig. 3, with thicker red symbols marking the values of the maximum temperature, $80_{-30}^{+70} \mathrm{MK}$, and the temperature at the time of maximum density, $37_{-6}^{+9} \mathrm{MK}$. We note that these values are averages over the real temperature distribution of the flaring plasma, and do not directly correspond to the $T_{0}$ and $T_{\mathrm{M}}$ used in Eq. (1) that are the temperatures at the loop apex. The parameters $T_{0}$ and $T_{\mathrm{M}}$ can however be obtained from the measured temperatures with the transformation formulae reported in Reale (2007), yielding $T_{0}=210_{-80}^{+240} \mathrm{MK}$ and $T_{\mathrm{M}}=81_{-16}^{+24} \mathrm{MK}$.

From the above estimates of $t_{0}, t_{\mathrm{M}}, T_{0}$, and $T_{\mathrm{M}}$, we finally derived two estimates of the length of the flaring loop $L$ with the formulae in Eq. (1). To estimate the uncertainties in $L$, we performed Monte Carlo simulations ${ }^{3}$ of the distribution of the input parameters $t_{0}, t_{\mathrm{M}}, T_{0}$, and $T_{\mathrm{M}}$, and inferred the $L$ quantiles corresponding to $\pm 1 \sigma$. The uncertainties in $L$ are dominated by those in $T_{0}$ and $T_{\mathrm{M}}$. In particular, the poorly constrained upper limit to $T_{0}$ yields a poorly constrained upper limit also on $L$. Both expressions for $L$ provide very similar results, supporting the validity of the analysis. The best values of $L$ are 10-20 $R_{\odot}$, with a lower limit at $6 R_{\odot}$, and a virtually unconstrained upper limit. Summarizing we found that the flare occurs in a large-scale magnetic loop with half length $L \sim 10-20 R_{\odot}=5-10 R_{\star}$.

We note that there is no evidence of flare occultation by the disk or by the star itself because of stellar rotation, and that there is no evidence of higher absorption during the flare. This indicates that during its evolution the flaring loop always remains observable and that no significant amount of infalling material passes along the line of sight.

\subsection{Dispersed spectra analysis and long-term variability}

The dispersed HEG and MEG spectra give access to individual line fluxes and allow us to derive detailed information about the $\mathrm{X}$-ray emitting plasma, such as the emission measure distribution $(E M D)$, the element abundances, and the plasma density. The EMD is a powerful tool to monitor the amount of plasma in small temperature bins and can only be derived from individual emission lines, not measurable in low resolution X-ray spectra. We measured line fluxes and performed our complete spectral analysis separately for the spectra gathered in each of the two observing segments. We can therefore explore, with the details offered by high resolution spectroscopy, variability on timescales of $\sim 100 \mathrm{ks}$. Shorter timescales cannot be investigated with these diagnostic tools because of the limited $S / N$. We also analyzed the spectrum collected during the entire observation to obtain time-averaged plasma properties with the highest possible accuracy.

To increase the $S / N$, we coadded the MEG and HEG first order spectra, after rebinning the HEG spectra to the MEG wavelength grid. With this procedure, we obtained two dispersed spectra, one for each of the two observing segments,

\footnotetext{
3 This procedure was chosen instead of simple error propagation that requires uncertainties to be small, because this is not the case for our two temperatures.
} 


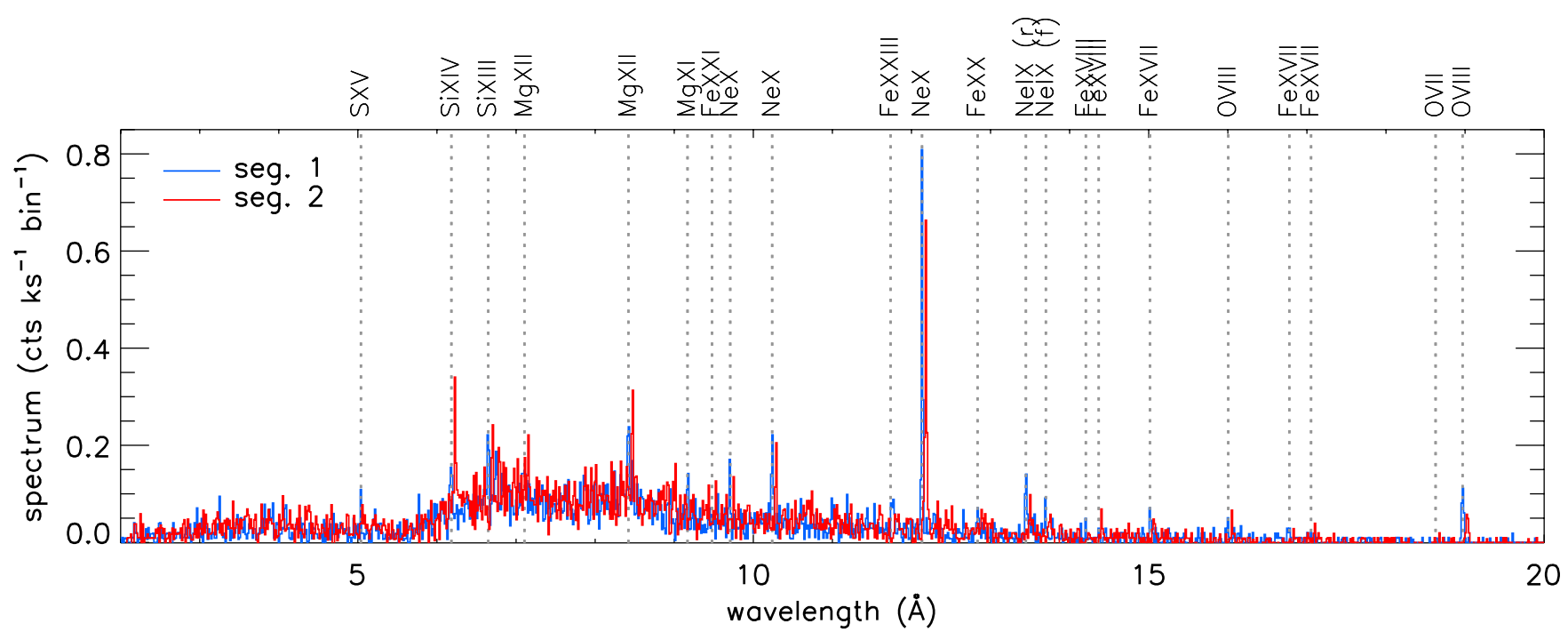

Fig. 4. V2129 Oph X-ray spectra observed with Chandra (HEG+MEG first orders) during the two observing segments. For clarity reasons, both spectra are rebinned by a factor of three (bin size $=0.015 \AA$ ), and the spectrum of seg. 2 is shifted toward longer wavelengths by $0.05 \AA$.

totaling 3750 and 4274 net counts, respectively. Figure 4 shows the resulting spectra with labels identifying the strongest emission lines. The subsequent analysis was performed using the IDL package PINTofALE v2.0 (Kashyap \& Drake 2000), and the CHIANTI v4.2 atomic database (Young et al. 2003).

\subsubsection{Flux measurements}

We measured the fluxes of the strongest emission lines in the observed X-ray spectra of V2129 Oph. Along with the line fluxes, we also measured continuum fluxes in selected wavelength intervals free of significant line contamination (see below).

Line fluxes were obtained by direct integration of the binned spectra. For the integration, we considered wavelength intervals centered around the predicted wavelength of each line $e^{4}$. For each line, we subtracted the continuum contribution, estimated from nearby wavelength intervals free of clearly evident emission lines, and corrected the resulting flux for the fraction of the line-profile, assumed to be Gaussian, outside the integration domain. Effective areas and exposure times were finally used to convert the observed line counts into fluxes. The observed net fluxes are listed, for each observing segment and the entire observation, in Table 1. For each line, we indicate the ion generating it; line blends are indicated by listing the different ions contributing to the emission at that wavelength. We also list the temperature of maximum formation, $T_{\max }$, of each measured line (for line blends $T_{\max }$ is obtained from the sum of the emissivities of the blended lines). The temperature $T_{\max }$ ranges between 2 and $16 \mathrm{MK}$. This range of maximum formation temperatures indicates that there is a significant amount of plasma at a few MK, a result that did not emerge from the analysis of the time-averaged low-resolution zeroth order spectrum in Sect. 3.2, or the analysis of the zeroth and heavily rebinned first order spectra in Sect. 3.3. In addition to the strong lines, we also included in Table 1 other well known lines, even if their fluxes are compatible with zero; upper limits are useful constraints of the EMD reconstruction.

\footnotetext{
${ }^{4}$ Given the discrete nature of the energy binning and the need to sum over an integer number of bins, the full width of the integration intervals is line-dependent, and ranges between 3 and 5 wavelength bins, corresponding to 2.2 and $3.4 \sigma$ of the line profiles.
}

In addition to line fluxes, we also measured continuum fluxes in selected wavelength ranges. These measurements are useful to constrain: a) the source metallicity ${ }^{5}$ and b) and the $E M$ of the hottest plasma components ${ }^{6}$. We measured the continuum emission of V2129 Oph in wavelength intervals free of significant line contributions (in particular, we selected intervals in which the expected line contribution with respect to the continuum is below $10 \%$, assuming solar metallicities and for any temperature ranging between $10^{4}$ and $10^{8} \mathrm{~K}$ ). The continuum emission in these wavelength intervals was estimated by integrating the observed counts, and subtracting the background contribution. The selected intervals and the corresponding measured fluxes are listed in Table 1.

Figure 5 shows a comparison of the measured fluxes (of both lines and continuum) in the two observing segments. We only plot, as a function of wavelength, the measured fluxes detected at the $2 \sigma$ level in at least one segment. Fluxes at short wavelengths tend to be, on average, higher in seg. 2 than in seg. 1, while the opposite is true for fluxes at long wavelengths (these differences are also marginally observable in the plot of the two spectra, Fig. 4). In many cases, this difference is significant at the $1 \sigma$ level. The analysis of the low resolution spectra has excluded there being any significant variability in the absorption, hence we can reasonably ascribe differences in line fluxes to differences in the $E M D$.

The two coolest lines detected at the $2 \sigma$ level in at least one segment are the O VIII Ly $\alpha$ line at $18.97 \AA$ and the Ne IX resonance line at $13.45 \AA$, mostly emitted by plasma at 3-4 MK. These lines show flux differences between the two segments of 1.7 and $1.5 \sigma$, with seg. 1 fluxes higher than those of seg. 2 . Combining the two differences, we obtain a very low probability $(0.3 \%)$ that the $E M$ of the plasma at $3-4 \mathrm{MK}$ producing these lines has not changed. Continuum fluxes that probe variations in the hottest plasma components, are systematically higher during

${ }^{5}$ Line fluxes are proportional to abundances, while the continuum is not. The relative strength of the line and continuum emission is therefore a measurement of the plasma abundances.

6 The major contributor to the continuum emission is bremsstrahlung, whose emissivity increases for increasing temperatures. The continuum emission level is therefore highly sensitive to the hottest plasma components. 
A\&A 530, A1 (2011)

Table 1. Measured fluxes of emission lines and continuum intervals in the X-ray spectrum of V2129 Oph.

\begin{tabular}{|c|c|c|c|c|c|}
\hline$\lambda^{a}$ & Ion & $\log T_{\max }^{b}$ & $\begin{array}{l}\text { seg. } 1 \\
\text { flux }^{c}\end{array}$ & $\begin{array}{l}\text { seg. } 2 \\
\text { flux }^{c}\end{array}$ & $\begin{array}{l}\text { seg. } 1+2 \\
\text { flux }^{c}\end{array}$ \\
\hline & & Lines & & & \\
\hline 5.04 & $\mathrm{Sxv}$ & 7.20 & $1.2 \pm 0.7$ & $0.2 \pm 0.6$ & $0.7 \pm 0.4$ \\
\hline 6.18 & Si xIV Si XIV & 7.20 & $0.8 \pm 0.3$ & $1.3 \pm 0.4$ & $1.1 \pm 0.3$ \\
\hline 6.65 & Si XIII & 7.00 & $0.9 \pm 0.4$ & $1.0 \pm 0.4$ & $1.0 \pm 0.3$ \\
\hline 6.69 & Si xIII Si XIII & 6.95 & $0.0 \pm 0.3$ & $0.1 \pm 0.3$ & $0.0 \pm 0.2$ \\
\hline 6.74 & Si XIII & 7.00 & $0.9 \pm 0.4$ & $1.0 \pm 0.3$ & $0.9 \pm 0.2$ \\
\hline 7.11 & $\operatorname{Mg}$ XII $M g$ XII & 7.00 & $0.3 \pm 0.3$ & $0.7 \pm 0.3$ & $0.5 \pm 0.2$ \\
\hline 8.42 & Mg XII Mg XII & 7.00 & $1.1 \pm 0.4$ & $1.5 \pm 0.4$ & $1.3 \pm 0.3$ \\
\hline 9.17 & $\operatorname{Mg} x I$ & 6.80 & $0.9 \pm 0.5$ & $0.7 \pm 0.5$ & $0.8 \pm 0.3$ \\
\hline 9.23 & $\mathrm{Mg}$ XI & 6.80 & $0.5 \pm 0.5$ & $0.0 \pm 0.3$ & $0.1 \pm 0.3$ \\
\hline 9.31 & $\operatorname{Mg}$ XI & 6.80 & $0.0 \pm 0.4$ & $0.3 \pm 0.4$ & $0.2 \pm 0.3$ \\
\hline 9.48 & Nex Nex Fe xxi & 7.00 & $0.2 \pm 0.4$ & $0.8 \pm 0.5$ & $0.5 \pm 0.3$ \\
\hline 9.71 & $\mathrm{Ne} x \mathrm{Ne} x$ & 6.80 & $1.2 \pm 0.6$ & $0.9 \pm 0.5$ & $1.0 \pm 0.4$ \\
\hline 10.24 & $\mathrm{Ne} x \mathrm{Ne} x$ & 6.80 & $2.5 \pm 0.7$ & $2.7 \pm 0.7$ & $2.6 \pm 0.5$ \\
\hline 11.74 & Fe xxIII & 7.20 & $0.0 \pm 0.6$ & $0.8 \pm 0.7$ & $0.4 \pm 0.4$ \\
\hline 11.77 & Fe XxII & 7.10 & $1.6 \pm 0.9$ & $0.6 \pm 0.7$ & $1.1 \pm 0.5$ \\
\hline 12.13 & $\mathrm{Ne} x \mathrm{Ne} x$ & 6.80 & $19.9 \pm 2.4$ & $15.7 \pm 2.1$ & $17.8 \pm 1.5$ \\
\hline 12.26 & Fe xvII & 6.80 & $0.5 \pm 0.8$ & $0.9 \pm 0.8$ & $0.7 \pm 0.5$ \\
\hline 12.28 & Fe xxI & 7.00 & $1.1 \pm 0.9$ & $1.6 \pm 0.9$ & $1.4 \pm 0.6$ \\
\hline 12.83 & Fe $x x$ Fe $x x$ & 7.00 & $1.3 \pm 1.2$ & $1.4 \pm 1.2$ & $1.3 \pm 0.8$ \\
\hline 13.45 & Ne IX & 6.60 & $8.1 \pm 2.4$ & $3.6 \pm 1.8$ & $5.8 \pm 1.4$ \\
\hline 13.55 & $\mathrm{Ne}$ IX & 6.55 & $2.3 \pm 1.7$ & $0.3 \pm 1.2$ & $1.3 \pm 0.9$ \\
\hline 13.70 & $\mathrm{Ne}$ IX & 6.60 & $2.8 \pm 1.8$ & $2.8 \pm 1.7$ & $2.8 \pm 1.1$ \\
\hline 14.20 & Fe xvIII Fe xvIII & 6.90 & $2.2 \pm 2.0$ & $0.6 \pm 1.6$ & $1.4 \pm 1.1$ \\
\hline 14.37 & Fe xviII Fe xvıII Fe xviII & 6.90 & $1.2 \pm 1.6$ & $1.9 \pm 1.7$ & $1.6 \pm 1.0$ \\
\hline 15.02 & Fe xvII & 6.75 & $5.5 \pm 2.8$ & $3.7 \pm 2.4$ & $4.6 \pm 1.7$ \\
\hline 16.01 & Fe xvIII O vIII O vIII & 6.50 & $3.9 \pm 3.1$ & $7.0 \pm 3.5$ & $5.5 \pm 2.1$ \\
\hline 16.78 & Fe xvII & 6.70 & $4.8 \pm 3.7$ & $2.3 \pm 3.1$ & $3.5 \pm 2.1$ \\
\hline 17.05 & Fe xVII & 6.70 & $3.0 \pm 3.7$ & $5.0 \pm 4.1$ & $4.0 \pm 2.4$ \\
\hline 17.10 & Fe xvII & 6.70 & $1.7 \pm 3.4$ & $3.8 \pm 3.9$ & $2.8 \pm 2.2$ \\
\hline 18.63 & O viI & 6.35 & $1.5 \pm 4.5$ & $3.4 \pm 5.0$ & $2.4 \pm 2.8$ \\
\hline 18.97 & O viII O vIII & 6.50 & $45.3 \pm 13.2$ & $18.3 \pm 9.3$ & $31.6 \pm 7.5$ \\
\hline & & Continuum & & & \\
\hline$[2.01 \div 2.51]$ & & & $4.9 \pm 1.0$ & $6.6 \pm 1.1$ & $5.8 \pm 0.7$ \\
\hline$[2.51 \div 2.99]$ & & & $5.2 \pm 0.8$ & $5.7 \pm 0.9$ & $5.5 \pm 0.6$ \\
\hline$[3.38 \div 3.64]$ & & & $3.8 \pm 0.7$ & $5.7 \pm 0.8$ & $4.8 \pm 0.5$ \\
\hline$[3.78 \div 3.88]$ & & & $2.7 \pm 0.6$ & $2.8 \pm 0.6$ & $2.7 \pm 0.4$ \\
\hline$[4.33 \div 4.67]$ & & & $5.6 \pm 1.1$ & $8.7 \pm 1.3$ & $7.2 \pm 0.8$ \\
\hline$[6.29 \div 6.53]$ & & & $5.4 \pm 0.6$ & $6.5 \pm 0.6$ & $5.9 \pm 0.4$ \\
\hline
\end{tabular}

Notes. ${ }^{(a)}$ Wavelengths $(\AA) ;{ }^{(b)}$ temperature $(\mathrm{K})$ of maximum emissivity; ${ }^{(c)}$ observed fluxes $\left(10^{-6} \mathrm{ph} \mathrm{s}^{-1} \mathrm{~cm}^{-2}\right)$ with uncertainties at the $68 \%$ confidence level.

seg. 2 than seg. 1, firmly indicating that the $E M$ at $T>10 \mathrm{MK}$ is higher during seg. 2.

\subsubsection{Emission measure distribution}

We derived the EMD and abundances of the plasma responsible for the X-ray emission of V2129 Oph by applying the Markov chain Monte Carlo method of Kashyap \& Drake (1998) to the measured continuum and line fluxes in Table 1. Measured fluxes were converted into unabsorbed fluxes by adopting the hydrogen column density $N_{\mathrm{H}}$ of $8 \times 10^{20} \mathrm{~cm}^{-2}$ derived from the zeroth order spectrum (see Sect. 3.2). For the EMD and abundance reconstruction, we considered all the measured fluxes discarding only the lines that depend on plasma density, i.e. the intercombination and forbidden lines of He-like triplets.

Starting from the flux measurements and considering the peak temperature at which each line is produced (see Table 1), we reconstructed the EMD over a regular logarithmic temperature grid with 0.15 dex bins in the $\log T$ range between 6.2 and 7.5. Abundances were estimated for those elements for which at least one line was measured. We note that the reconstructed $E M$ values associated with bins at the boundary of the chosen temperature domain may be overestimated: artificially limiting the temperature range of the $E M D$ reconstruction forces to zero the EMD outside this range. Plasma at higher and lower temperatures may however contribute to some of the measured fluxes, thus causing the $E M$ values at the boundary bins to be overestimated to account for the observed fluxes.

The EMD and abundances derived from the whole observation are reported in Table 2 and the EMD is also plotted in Fig. 6. Figure 6 also shows the two-temperature (2-T) model obtained from the zeroth order spectrum of the whole observation (see Sect. 3.2). We note that the two components of this 2- $T$ model agree very well with the two hottest peaks of the EMD.

We then derived EMD and abundances of the emitting plasma by separately considering the two observing segments. For both segments, unabsorbed fluxes were evaluated assuming $N_{\mathrm{H}}=8 \times 10^{20} \mathrm{~cm}^{-2}$, because no evidence of absorption variability was found (see Sect. 3.3.2). No significant variation in plasma 


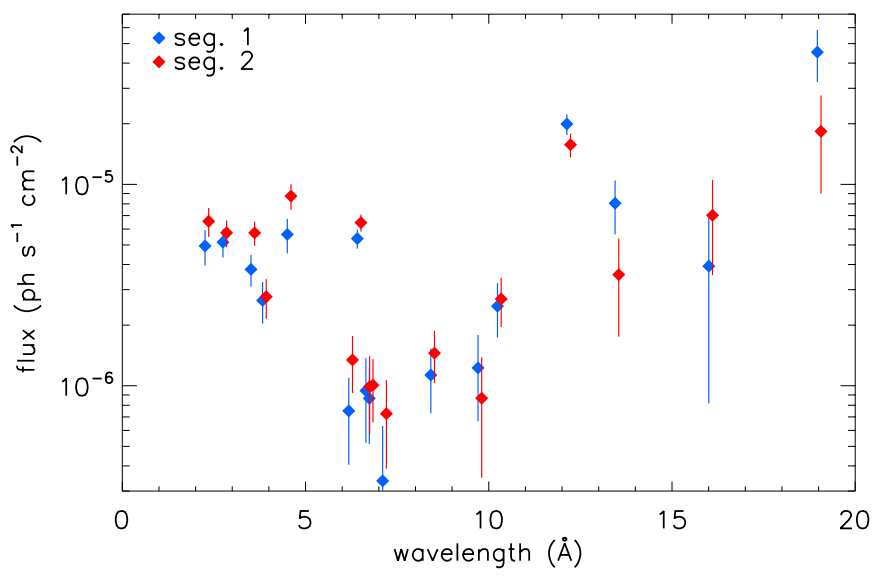

Fig. 5. Comparison of measured continuum and lines fluxes in the two observing segments. Only features detected at the $2 \sigma$ level in at least one segment are plotted.

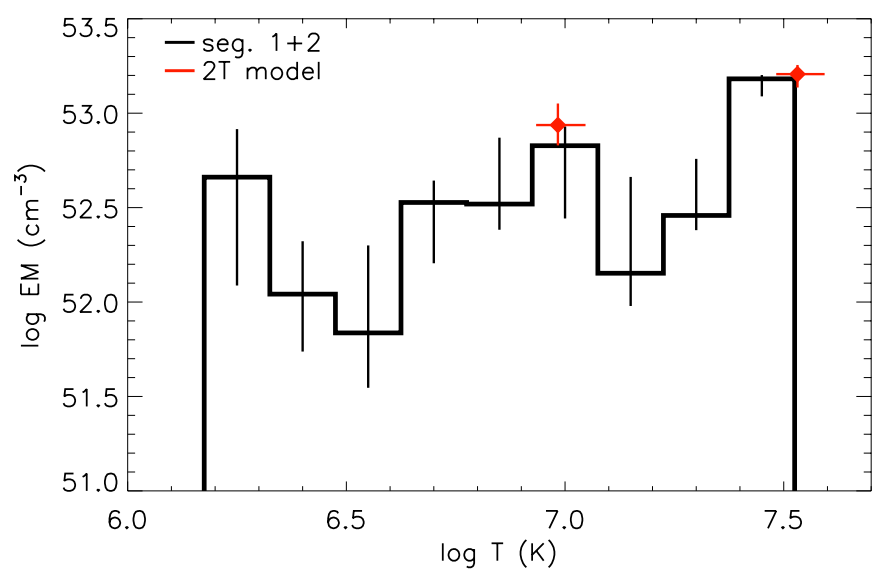

Fig. 6. $E M D$ obtained from the flux measurements of the whole observation (seg. 1+2).

abundances can be discerned between seg. 1 and seg. 2, indicating that the observed variations in line fluxes can be ascribed to $E M D$ variations only. We therefore repeated the $E M D$ reconstruction for seg. 1 and seg. 2 freezing the plasma abundances to the values of the seg. $1+2$ model, and leaving the $E M$ values as free parameters. The $E M D$ derived in this way for the two segments are reported in Table 2, and plotted in Fig. 7. As a consequence of the observed systematic differences between the measured line fluxes, a difference is seen in the cool plasma component ${ }^{7}$, such that $E M D_{1}>E M D_{2}$ at the $1.5 \sigma$ level in the bin centered on $\log T=6.55(T \sim 3-4 \mathrm{MK})$. We note that the significance of the time variability is lower for the EMD than the line fluxes (see Sect. 3.4.1) because the EMD reconstruction procedure involves further uncertainty sources (Kashyap \& Drake 1998). However, the high confidence level inferred for the variation in the flux of cool lines indicates that the EMD variability is indeed real.

\footnotetext{
${ }^{7}$ Note that the corresponding models obtained without fixed abundances, have $E M D$ values almost identical to those obtained by fixing them, but the associated $E M D$ uncertainties are larger and the differences at low temperatures are not significant.
}

Table 2. EMD and abundances of V2129 Oph.

\begin{tabular}{cccc}
\hline \hline $\log T(\mathrm{~K})$ & $\begin{array}{c}\text { seg. 1 } \\
\log E M\end{array}$ & $\begin{array}{c}\text { seg. } 2 \\
\log E M\end{array}$ & $\begin{array}{c}\text { seg. } 1+2 \\
\log E M\end{array}$ \\
\hline 6.25 & $52.67_{-0.77}^{+0.30}$ & $52.60_{-1.29}^{+0.21}$ & $52.66_{-0.57}^{+0.25}$ \\
6.40 & $52.01_{-0.40}^{+0.31}$ & $51.83_{-0.25}^{+0.29}$ & $52.04_{-0.30}^{+0.28}$ \\
6.55 & $52.48_{-0.38}^{+0.18}$ & $51.28_{-0.19}^{+0.67}$ & $51.84_{-0.29}^{+0.46}$ \\
6.70 & $52.16_{-0.13}^{+0.46}$ & $51.83_{-0.22}^{+0.50}$ & $52.53_{-0.32}^{+0.12}$ \\
6.85 & $52.91_{-0.31}^{+0.07}$ & $52.63_{-0.54}^{+0.13}$ & $52.52_{-0.14}^{+0.35}$ \\
7.00 & $52.68_{-0.27}^{+0.15}$ & $52.84_{-0.37}^{+0.10}$ & $52.83_{-0.39}^{+0.10}$ \\
7.15 & $52.06_{-0.16}^{+0.16}$ & $52.31_{-0.18}^{+0.43}$ & $52.15_{-0.17}^{+0.51}$ \\
7.30 & $52.37_{-0.09}^{+0.36}$ & $52.34_{-0.03}^{+0.52}$ & $52.46_{-0.08}^{+0.30}$ \\
7.45 & $53.11_{-0.11}^{+0.03}$ & $53.24_{-0.12}^{+0.02}$ & $53.18_{-0.09}^{+0.02}$ \\
\hline \multicolumn{5}{c}{ Abundances } \\
$\mathrm{O}=0.61_{-0.21}^{+0.21}$ & $\mathrm{Ne}=1.09_{-0.26}^{+0.29}$ & $\mathrm{Mg}=0.15_{-0.04}^{+0.04}$ \\
$\mathrm{Si}=0.15_{-0.04}^{+0.03} \mathrm{~S}=0.38_{-0.32}^{+0.14}$ & $\mathrm{Fe}=0.13_{-0.04}^{+0.04}$ \\
\hline \multicolumn{5}{c}{}
\end{tabular}

Notes. Abundances are in solar units (Asplund et al. 2005). EMD values are in $\mathrm{cm}^{-3}$ and have been derived assuming that V2129 Oph is located at $120 \mathrm{pc}$.

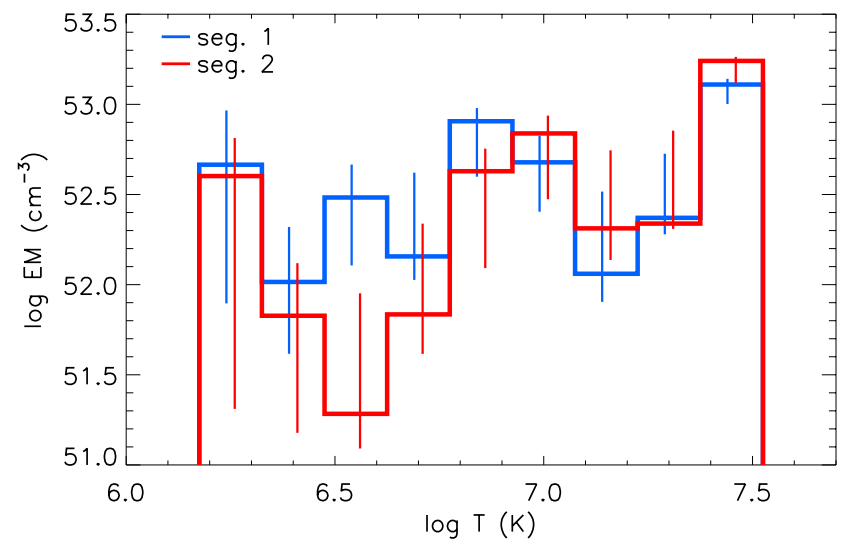

Fig. 7. $E M D$ obtained from the flux measurements of the two observing segments.

\subsubsection{Electron density}

The plasma density $N_{\mathrm{e}}$ can be constrained, from the high resolution X-ray spectroscopy, by analyzing the He-like line triplets, and, in particular, the flux ratio of the intercombination line, $i$, to the forbidden line, $f$ (Gabriel \& Jordan 1969). Increasing the plasma densities leads to a decrease in the $f / i$ ratios. The intensities of the $i$ and $f$ lines depend on plasma density because these lines originate from the radiative decay of metastable states (long lifetime states), whose depopulation is enhanced by increasing the collision rate (i.e. increasing the density), while the resonance lines originate from excited states depopulated only by photon emission processes.

Among the He-like triplets in the wavelength range covered by the Chandra/HETGS instrument, we identified and measured the He-like line triplets of Si XIII (at 6.65, 6.69, 6.74 $\AA$ ), Mg XI (at 9.17, 9.23, 9.31 $\AA$ ), and $\mathrm{Ne}$ IX (at 13.45, 13.55, $13.70 \AA$ ). The analysis of these triplets can constrain the electron density of plasma at the formation temperatures of these ions, $\sim 10, \sim 6$, and $\sim 4 \mathrm{MK}$, respectively. The measured line fluxes of these triplets are reported in Table 1.

The Si XIII triplet has, in all of our spectra, $i$ line fluxes compatible with zero within $1 \sigma$, providing therefore only a lower 


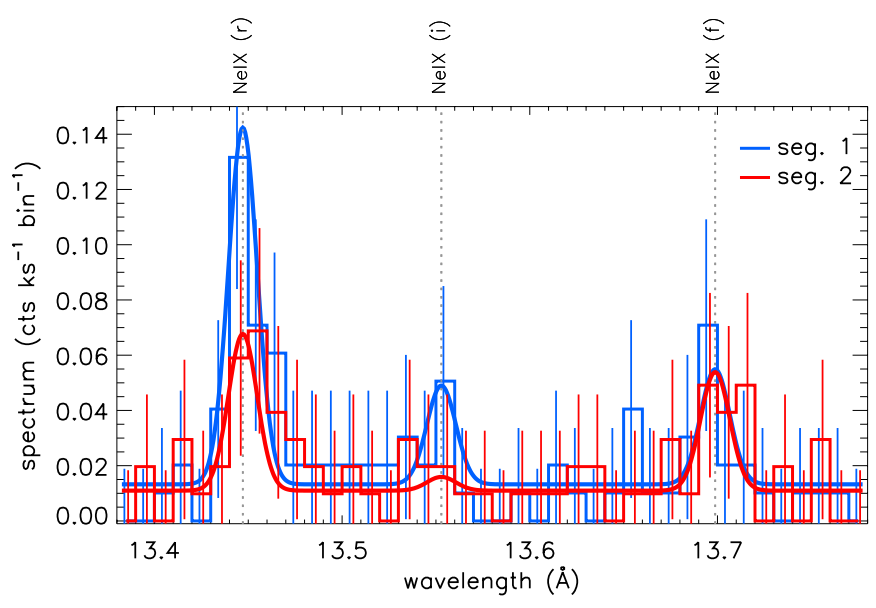

Fig. 8. Spectra of the two observing segments in the Ne Ix triplet region. Observed spectra are rebinned by a factor two (bin size $=0.01 \AA$ ). Model spectra are obtained using line and continuum measurements (see Sect. 3.4.1) and assuming a Gaussian line spread function.

limit to the $f / i$ ratio. We therefore infer that plasma at $T \sim$ $10 \mathrm{MK}$ has a density lower than $\sim 10^{12} \mathrm{~cm}^{-3}$, during both seg. 1 and seg. 2. The Mg xi triplet, mostly emitted by plasma at $6 \mathrm{MK}$, has both $i$ and $f$ line fluxes compatible with zero in all the spectra, thus provides no constraint on the plasma density.

In Fig. 8, we show the lines of the Ne Ix triplet corresponding to the two observing segments. These lines are produced by plasma at $\sim 4 \mathrm{MK}$. In the plot, we also show noise-free spectra reconstructed on the basis of the measured continuum and line counts. The overall flux of the triplet is clearly lower in the second observing segment than in the first (see also Table 1).

To obtain an accurate estimate of the $f / i$ ratio uncertainties, we considered the measured counts of the $i$ and $f$ lines, as well as those used to determine the continuum level. Assuming that these quantities follow Poisson distributions, we derived the distribution of the $f / i$ flux ratio using Monte Carlo simulations, and hence inferred the $f / i$ mean value and the quantiles corresponding to $\pm 1 \sigma$. With this procedure and after correcting for interstellar absorption, we obtained $f / i=1.2_{-0.6}^{+1.6}$ and $f / i>2.3$ for seg. 1 and seg. 2 , respectively. A comparison of the observed $f / i$ values with the predicted $f / i$ - -atio $^{8}$ vs. plasma density is given in Fig. 9. From this relation, we infer that $\log N_{\mathrm{e}}=12.1_{-1.0}^{+0.5}$ and $\log N_{\mathrm{e}}<11.5$ for seg. 1 and seg. 2, respectively, where $N_{\mathrm{e}}$ is in $\mathrm{cm}^{-3}$.

\section{Main results}

The Chandra observation of V2129 Oph has revealed that it is an X-ray bright source. Its average X-ray luminosity, derived from our mean $E M D$, is $L_{X}=2.7 \times 10^{30} \mathrm{erg} \mathrm{s}^{-1}$ in the $0.5-10.0 \mathrm{keV}$ band. This value of $L_{\mathrm{X}}$, combined with the bolometric luminosity of V2129 Oph $\left(\log L_{\text {bol }} / L_{\odot}=0.15\right.$, Donati et al. 2011), implies that $\log L_{\mathrm{X}} / L_{\mathrm{bol}}=-3.3$, a value that puts V2129 Oph among the X-ray brightest CTTS when compared to CTTS in the ONC (Preibisch et al. 2005). The X-ray emission from V2129 Oph is produced by significant amounts of plasma spanning a broad temperature range, from at least as low

\footnotetext{
8 The predicted ratios depend very weakly also on the plasma $E M D$, other than on density. In deriving the $f / i$-ratio vs. density relation, we adopted the mean $E M D$ for the whole observation. Using the individual $E M D$ derived for the two segments, however, produces negligible differences in the inferred $\log N_{\mathrm{e}}$, smaller than 0.01 dex.
}

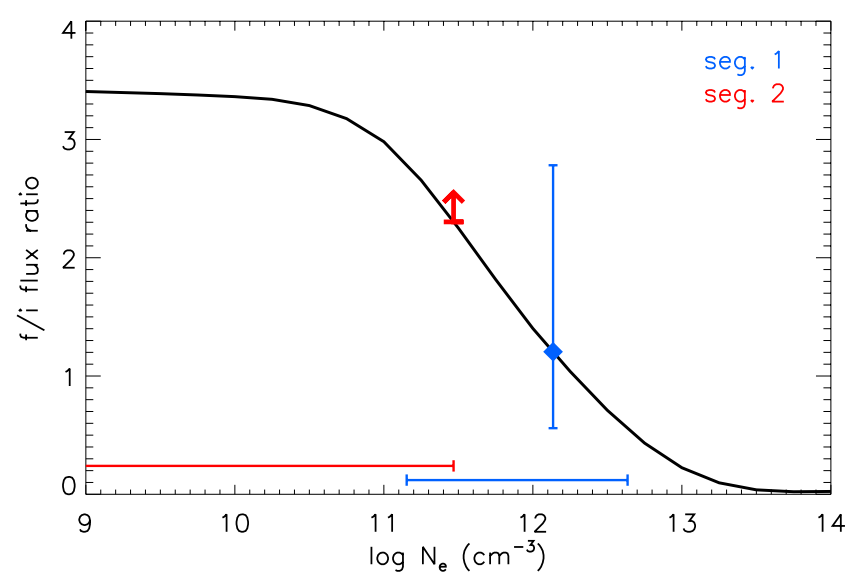

Fig. 9. Forbidden $f$ to intercombination $i$ line ratio of the Ne ix triplet vs. electron density. Black curve represents the $f / i$ predicted ratio. Observed $f / i$ values of seg. 1 and seg. 2, and the corresponding density ranges, are indicated in blue and red, respectively.

as $2 \mathrm{MK}$ to $\sim 30 \mathrm{MK}$, as indicated by the reconstructed $E M D$ (see Fig. 6). In particular, the plasma at a few MK, monitored during the first half of the Chandra observation, shows high density, $\log N_{\mathrm{e}} \approx 12.1$, which is consistent with results for almost all the other CTTS with measured densities, supporting the idea that the presence of high density cool plasma is directly linked to the accretion phenomenon.

\subsection{X-ray variability}

The X-ray emission of V2129 Oph is variable on short timescales (of a few hours). In the 200 ks Chandra observation, we detected a clear flare, $\sim 10 \mathrm{~h}$ long, causing an increase in the $\mathrm{X}$-ray luminosity by a factor of $\sim 3$. This flare occurred in a long loop, with an analysis of the rise phase giving a best value of the loop half length of $\sim 5-10 R_{\star}$, with a lower limit of $3 R_{\star}$ and an unconstrained upper limit.

Beside this clear flare, all the observation is characterized by variability similar to that typically produced by coronal activity, i.e. with a clearly correlated X-ray emission level and spectral hardness. This short-term variability is mainly produced by the hottest $(T \approx 34 \mathrm{MK}$ ) plasma components.

The analysis of the high resolution spectra collected during the two segments allowed us to search for any variability in the X-ray emission observed at two different phases during stellar rotation and, therefore, emerging from either different magnetospheric structures (corona and accretion shock) or from the same structures seen with different viewing geometries. We stress that the analysis of the high resolution spectra allows us to probe the coolest plasma components. This comparison yielded two main results.

1. Cool plasma EM variability. Lines at long wavelengths were significantly enhanced during seg. 1 with respect to seg. 2. This finding is apparently not related to any variation in the absorbing column since no such variability is derived from the XSPEC analysis of the heavily rebinned zeroth + first order spectra. The EMD of the two observing segments indeed indicates that this difference originates from a difference in the amount of observable emission measure at low temperatures. In particular, we observe a difference in the $E M$ at $T \approx 3-4 \mathrm{MK}(\log T=6.55)$, where $E M D_{1}$ is higher than $E M D_{2}$, at the $1.5 \sigma$ level, by more than a factor of ten. The difference in the amount of emission measure at 
a few MK is likely not related to flaring activity, since, as indicated by the short timescale analysis, flares contribute to the EMD at much hotter temperatures than $4 \mathrm{MK}$ (see Fig. 2).

2. Plasma density variability. During the two observing segments, we found different constraints on the density of the plasma component at a few MK. In seg. 1, the cool plasma density can be constrained between $1.3 \times 10^{11}$ and $4 \times 10^{12} \mathrm{~cm}^{-3}$, while during seg. 2 the density diagnostic is compatible with a low density plasma, $N_{\mathrm{e}}<3 \times 10^{11} \mathrm{~cm}^{-3}$. Although the statistical significance of this difference is low, V2129 Oph is the first CTTS where apparent variations in the plasma density over timescales of $\sim 100 \mathrm{ks}$ have been observed.

\section{Discussion}

We now discuss the implications of our observational results for the X-ray emission of V2129 Oph, taking advantage of the constraints on the properties and geometry of accretion shock region and on the stellar magnetic field, obtained by us simultaneously with the X-ray observation (Donati et al. 2011).

The analysis of X-ray data allows us to derive the main properties of the coronal plasma and of the plasma heated in accretion shocks, as discussed below. These results will also provide constraints for future works dedicated to magnetic field extrapolation, designed to model the coronal plasma and the accretion stream structures.

\subsection{Coronal emission}

The broad-band X-ray luminosity of V2129 Oph is dominated by emission from hot plasma components, most likely of coronal origin. In 1991, the ROSAT satellite detected V2129 Oph (Casanova et al. 1995) with an X-ray luminosity of $2.5 \times$ $10^{30} \mathrm{erg} \mathrm{s}^{-1}$, which is almost identical to that measured with Chandra in 2009. V2129 Oph thus appears to have a stable X-ray emission, hence coronal activity level, over timescales of $\sim 20 \mathrm{yr}$.

V2129 Oph displays X-ray variability over short timescales (Fig. 1). This variability, being mainly caused by very hot plasma, may be related to coronal activity. The X-ray light curve of the first observing segment is characterized by an obvious flare. We constrained the half length of the flaring loop, deriving 5-10 $R_{\star}$ as best value (with a lower limit of $3 R_{\star}$ and an unconstrained upper limit). Considering its length and the inner disk radius $\left(\sim 7.2 R_{\star}\right)$, we cannot exclude the possibility that this loop has one footpoint anchored to the inner circumstellar disk. These flaring coronal structures connecting star and inner disk were already suggested in young stars of the ONC (Favata et al. 2005; Getman et al. 2008), although we note that the presence of a disk is not a prerequisite for such large flaring loops to exist. They are also found to occur in stars that display no evidence of disks (Getman et al. 2008; Aarnio et al. 2010).

The second segment of the Chandra observation shows a trend in the first part of the segment that might be interpreted as the decay phase of a flare, followed in the second part by an increase in the count rate and the hardness ratio that might be associated with the rise phase of another flare.

Alternatively, the variability might be due to modulation of some emission feature on the stellar surface as the star rotates. We note that the observed dip in the light curve coincides with the passage along the line of sight of the large region of strong positive radial magnetic field (in red in Fig. 1), which is also coincident with an extended dark photospheric spot (not shown, cf. Donati et al. 2011). Although we can only speculate that the dip in the X-ray light curve is associated with the passage of this region, it is not unconceivable that the X-ray emitting corona, assumed to be confined by relatively small-scale closed magnetic loops, is disrupted in this region of strong magnetic field likely connecting the star to the inner disk (as is certainly true for the field in the accretion spot that lies within the region). Figure 2 might indicate that while this region removes a significant fraction of the visible hot (34 MK) plasma, it leaves the cool (9.6 MK) plasma relatively unaffected thus implying that the two plasma components are not co-spatial.

\subsection{High density cool plasma and accretion shock(s)}

During the first segment of our Chandra observation, we found that the plasma at a few MK has a high density $\left(\log N_{\mathrm{e}}=12.1\right)$. Therefore, V2129 Oph is another example of a CTTS in which cool plasma has high density. This plasma component is intrinsically different from the coronal plasma observed in nonaccreting active stars. The commonly accepted model suggests that this plasma component is material heated in the accretion shock. The high infall velocity of the accreting material naturally justifies material at these temperatures, of a few MK, and densities.

Plasma heated in accretion shocks is located at the base of the accretion stream, just behind the shock surface. Models predict that this accreted material, passing through the shock surface, strongly reduces its infall velocity, but continues its downward motion across the stellar atmosphere, cooling down and radiating X-rays (Günther et al. 2007; Sacco et al. 2008; Orlando et al. 2010).

The association of the cool plasma component at $\sim 3-4 \mathrm{MK}$ of V2129 Oph with the accretion shock is greatly reinforced if we consider that this temperature is almost exactly what we expect for the post-shock plasma. The measured strength of the magnetic field of V2129 Oph implies that the inner disk is truncated at 7.2 $R_{\star}$ (Donati et al. 2011). A free fall from that distance implies a pre-shock velocity of $460 \mathrm{~km} \mathrm{~s}^{-1}$, and, as a consequence, a post-shock temperature of $3 \mathrm{MK}$.

We therefore assume that X-rays from V2129 Oph are emitted by two plasma components: a) the high density plasma component at 3-4 MK, heated in the accretion shock and hence located at the base of the accretion stream; and b) a low density corona with $T$ ranging from 2 up to a $\sim 30 \mathrm{MK}$. We attempt to interpret the time variability of the $E M$ at $\sim 3-4 \mathrm{MK}$ and, possibly, of the measured plasma density that we observed between our two observing segments in terms of the different viewing geometries of the accretion shock and funnel due to stellar rotation. Figure 10 displays a cartoon describing the system and the viewing geometry during the two observing segments.

From a theoretical point of view, it has been debated whether X-rays emitted by shock-heated plasma in CTTS are able to escape from the shock region, and hence be detected (Drake 2005; Sacco et al. 2010). Their escape probability depends on the height of the shock surface in the stellar atmosphere and on the direction of emission. X-rays emitted in the direction of the accretion stream could be absorbed by the pre-shock infalling material, depending on the accretion stream density and dimensions along the line of sight; X-rays emitted perpendicularly to the accretion funnel could be absorbed by the surrounding stellar atmosphere, depending on the sinking of the hot post shock in the stellar atmosphere (Drake 2005; Sacco et al. 2010). The amount of X-rays produced by the accretion shock and able to escape in the different directions may be estimated by means of 


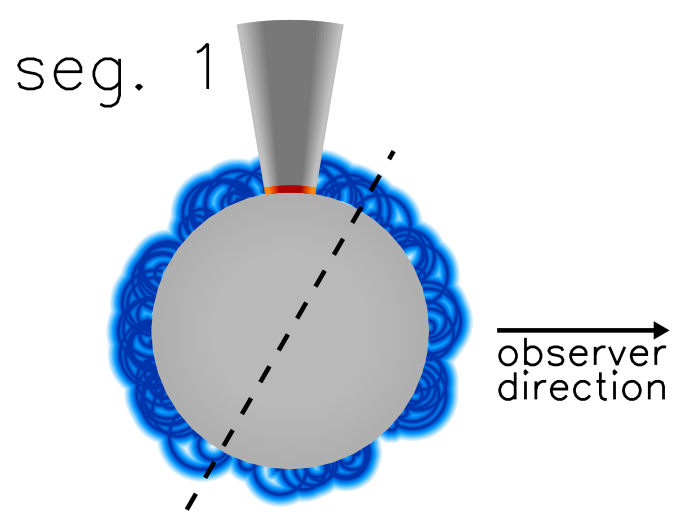

rot. axis

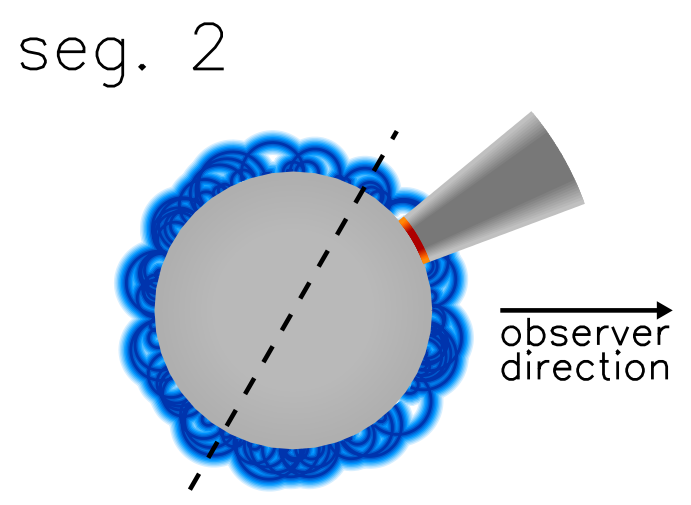

rot. axis
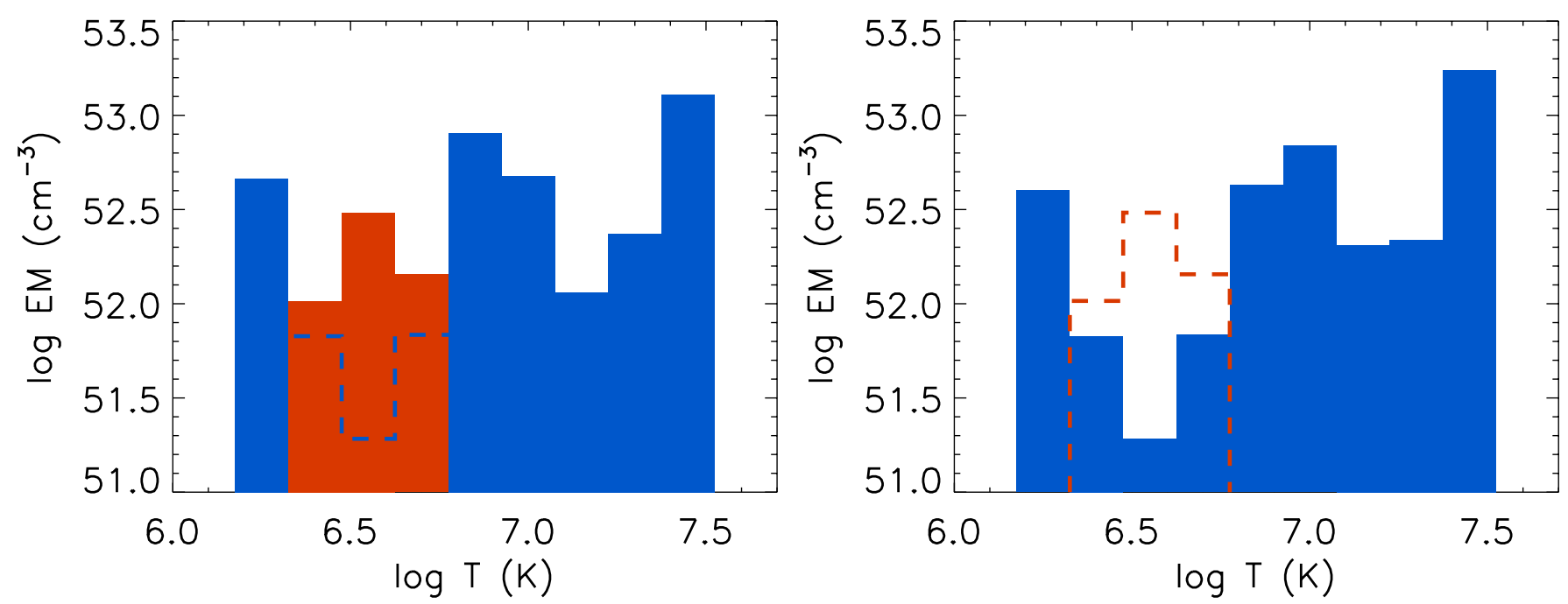

Fig. 10. Upper panels: cartoon describing the spatial distribution of the two X-ray emitting plasma components of V2129 Oph. Left and right cartoons correspond to the two viewing angles of the two Chandra observing segments, with observer in the rightward direction. Red region marks the post-shock high density plasma at 3-4 MK, blue regions indicate low density coronal plasma with $T$ ranging from 2 up to $\sim 30$ MK. Lower panels: $E M D$ corresponding to the two observing segments (see Sect. 3.4.2). Red $E M$ bins symbolize observed $E M$ values ascribed to post-shock plasma, while blue bins represent those accounting for coronal plasma. During seg. 1, the pre-shock material does not block the view of both post-shock and coronal plasma: the $E M D$ is therefore the superposition of the $E M D$ of the two components. During seg. 2, the pre-shock material almost completely absorbs the X-rays of the post-shock plasma emitted toward the observer, while coronal emission is mostly unaffected: in this case, all the X-rays detected are those produced by coronal plasma; the reconstructed $E M D$, being only those of coronal plasma, hence misses the high $E M$ values at 3-4 MK (reported for comparison with a dashed red line in the EMD plot).

detailed 3D modeling of the shock region, which is beyond the scope of this paper.

In the case of V2129 Oph, we can assume that the post-shock plasma is co-spatial with the optical hot spot, whose location we determined by performing Zeeman-Doppler imaging. As shown in Fig. 1 and outlined in the cartoon of Fig. 10, this region remains visible during both Chandra observing segments but with distinctly different viewing geometries: near the stellar limb during seg. 1 and nearer the center of the stellar disk during seg. 2.

During seg. 1, the accretion funnel is observed sideways. In this configuration, X-rays produced by the shock heated plasma can easily be observed because they can escape from the lateral part of the funnel. The accretion shock characteristics of V2129 Oph, i.e. a pre-shock velocity of $460 \mathrm{~km} \mathrm{~s}^{-1}$, a low metallicity, and a pre-shock density of $\sim 3 \times 10^{11} \mathrm{~cm}^{-3}$ (since the accretion shock is expected to be a strong shock, the density of the pre-shock material should be one fourth of the measured post-shock density), imply that the hot post-shock region is high enough above the stellar surface to avoid significant absorption from the surrounding chromosphere (Sacco et al. 2010). $\mathrm{X}$-rays from coronal plasma are of course also observable. The emerging X-ray spectrum and the resulting EMD are therefore the superposition of the two contributions, with the post-shock component dominating the EMD at 3-4 MK, and the corona dominating at higher temperatures. The observed X-ray spectrum of seg. 1 is indeed compatible with an overall low absorption, and with a high density for the plasma at $\sim 4 \mathrm{MK}$.

In contrast, during seg. 2 the hot spot almost faces the observer. Pre-shock infalling material is thus located between us and the post-shock region at the base of the accretion funnel (see Fig. 10). The X-rays emitted in our direction by post-shock plasma thus enter the pre-shock accretion stream. Even considering that our line of sight intersects a small fraction of the accretion funnel, i.e. $15 \%$ of the stellar radius, the resulting hydrogen column density is $\sim 7 \times 10^{21} \mathrm{~cm}^{-2}$ (having assumed a pre-shock density of $3 \times 10^{11} \mathrm{~cm}^{-3}$ ). This large column density 
would absorb $90 \%$ of X-rays at $\sim 13.5 \AA$ (the wavelength of the Ne Ix triplet). In this viewing geometry, essentially no X-ray emitted by the shock-heated plasma can be observed. Therefore during seg. 2 we can detect only coronal X-rays because they are unlikely to be blocked by the pre-shock material. The emerging $\mathrm{X}$-ray spectrum is therefore almost completely due to coronal plasma. The reconstructed EMD of seg. 2, hence misses the high $E M$ values at 3-4 MK, and the seg. 2 Ne Ix triplet reveals a low density plasma, compatible with a coronal origin. In this geometry, the emerging X-ray spectrum is again affected by the same low absorption as seg. 1.

We note that the lowest temperature bin of the EMD (at $2 \mathrm{MK}$ ), even if poorly constrained, appears constant between the two segments. This does not contradict the above picture for two reasons: 1) the error bars in the $E M$ might conceal even a large variation; and 2) HD simulations predict that accretion shocks onto CTTS produce sharply peaked EMD (Sacco et al. 2010) that might not contribute significantly to this $E M$ bin.

The proposed scenario is able to explain the observed variations in the EMD and density in terms of significant variations in the extinction suffered by the X-rays from the accretion shock. We note that this is compatible with the lack of variability in the absorption measured from the X-ray data. An increase in the X-ray absorption of the post-shock plasma cannot be detected only because of the presence of the underlying weakly absorbed $\mathrm{X}$-ray coronal emission.

In the optical band, the accretion indicators (e.g. the Ca II and He I lines) reach their maximum at phases $0.55-0.7$, corresponding to rotational phases at which the hot spot is directed toward us. We found the converse result for the accretion-driven X-ray emission, which reaches a maximum when the hot spot is near the stellar limb and a minimum when the hot-spot is facing us. We can reconcile these two opposite behaviors by considering that the infalling material is indeed almost completely in the gas phase. Gas is indeed transparent to optical radiation, so that the $\mathrm{Ca}$ II and $\mathrm{He}_{\mathrm{I}}$ emission can reach us through the funnel, but gas can easily absorb soft X-rays with energies $E<1 \mathrm{keV}$, such as those produced in the accretion shock.

We have argued that the plasma at 3-4 MK observed on V2129 Oph is likely to be shock-heated material. The high density, the time variability of its $E M$ in agreement with this picture, and the coincidence of the temperature with the expectation for the post-shock material reinforce this interpretation. In this likely hypothesis, we can infer the properties of the shockheated plasma, and in particular the accretion rate, needed to explain the observed soft X-ray flux. We consider the observed flux of the Ne Ix resonance line measured during seg. 1 and assume a pre-shock velocity of $460 \mathrm{~km} \mathrm{~s}^{-1}$ (compatible with a free fall from the estimated inner disk position) to derive an accretion rate of $7 \times 10^{-11} M_{\odot} \mathrm{yr}^{-1}$. This is lower by a factor of ten than the accretion rate derived from optical lines, $6 \times$ $10^{-10} M_{\odot} \mathrm{yr}^{-1}$ (Donati et al. 2011). This underestimation of $\dot{M}$ derived from X-rays is common for CTTS observed with high resolution X-ray spectroscopy (Argiroffi et al. 2009; Curran et al. 2011). As suggested by Günther et al. (2007) and investigated by (Sacco et al. 2010), this discrepancy could be explained by assuming that the accretion stream is not uniform in density and/or in velocity, hence that the portion of the accretion stream producing observable X-rays is a small fraction of the whole stream. Curran et al. (2011), compared mass accretion rates of CTTS derived from optical diagnostics with those derived from high resolution X-ray spectra, and argued that, even if X-ray data provide significantly underestimated values, a weak correlation

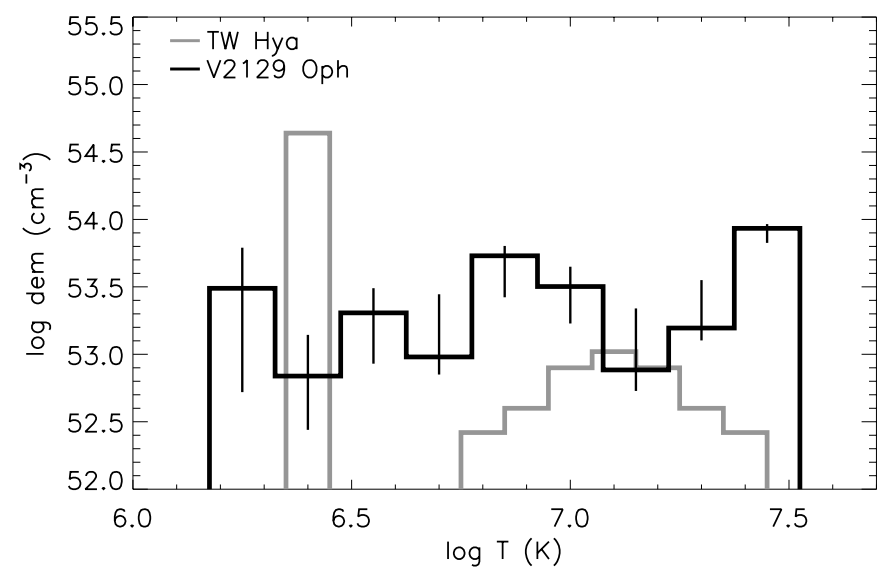

Fig. 11. Differential emission measure (dem) of V2129 Oph, compared to that of TW Hya derived by Brickhouse et al. (2010).

between the two quantities may exist. The values that we obtain for V2129 Oph support this tentative correlation.

Finally, having argued that, at least during seg. 1, the EMD bins at low temperature are caused by the accretionshock and those at high temperature by coronal plasma, we estimate separately the X-ray luminosities of the two components by assuming that the EMD below and above $\log T=6.8$ are entirely due, respectively, to shock-heated material and coronal plasma. From the EMD of seg. 1, the resulting $L_{X}$ are $3.4 \times 10^{29}$ and $2.2 \times 10^{30} \mathrm{erg} \mathrm{s}^{-1}$, for the shock-heated and the coronal plasma, respectively. Even when we do observe X-rays from shock-heated material (seg. 1), the coronal X-ray luminosity is thus $\sim 90 \%$ of the total $L_{\mathrm{X}}$.

A similar conclusion can be reached by comparing the $E M D$ reconstructed from individual line and continuum fluxes, with the two-temperature model obtained from the spectral fitting of the CCD-resolution ACIS spectrum (see Fig. 6). The temperatures in this latter model correspond to the two hottest peaks of the EMD distribution, both ascribable to coronal plasma. This is further confirmation that X-ray spectra of CTTS in the $0.5-10 \mathrm{keV}$ band are dominated by coronal emission. The shock-heated material is probed only by the softest part of the spectrum, or by emission lines produced by material at temperatures of few MK, which contribute little flux to the overall lowresolution spectra.

\subsection{V2129 Oph vs. TW Hya}

TW Hya is considered the prototype of CTTS with high-density $\mathrm{X}$-ray emitting plasma. TW Hya is certainly not the most representative star of its class, being among the oldest CTTS. Because of its proximity, however, it is one of the most widely studied, especially in the X-ray band (Kastner et al. 2002; Stelzer \& Schmitt 2004; Brickhouse et al. 2010).

We compare in Fig. 11 the differential emission measure $^{9}$ (dem) of V2129 Oph (relative to seg. 1) with that of TW Hya derived by Brickhouse et al. (2010).

The X-ray emission of TW Hya is dominated by plasma at $2.5 \mathrm{MK}$, as indicated by the sharp peak in the dem. This plasma component has very high density and is thus material heated in

9 The dem is defined as $E M D / \Delta \log T$. The use of the dem instead of the $E M D$ eases the comparison between models defined over temperature grids with different bin sizes since the $E M$ in a given temperature range can be simply evaluated as the area under the dem curve. 
the accretion shock. The high temperature tail ( $T \geq 10 \mathrm{MK})$ of the dem of TW Hya indicates instead that the amount of coronal plasma is significantly lower.

The thermal structure of the plasma on V2129 Oph differs significantly from that of TW Hya. The cool plasma on V2129 Oph has a much lower EM than that on TW Hya and V2129 Oph thus hosts a significantly smaller amount of high density cool plasma related to the accretion process. In contrast the corona of V2129 Oph is significantly more active than that of TW Hya, bearing a larger amount of emission measure at high temperatures. Apart from differing in terms of absolute values, we note that V2129 Oph and TW Hya have very different relative contributions of accretion-driven and coronal plasmas.

We note finally that the pole-on inclination under which TW Hya is viewed $(i \approx 7 \mathrm{deg}$, Qi et al. 2004) raises some questions about the observability of accretion-driven X-rays in terms of viewing geometry of the accretion shock. Postulating that TW Hya also has a hot spot latitude of $\sim 60 \mathrm{deg}$, then its accretion shock should be viewed at the same angle as the accretion shock of V2129 Oph during seg. 2. However, accretion-driven $\mathrm{X}$-rays from TW Hya are always observed. This difference could be reconciled by assuming for TW Hya a lower latitude of the accretion shock (as suggested by Batalha et al. 2002). Additional observational constraints on the magnetospheric accretion geometry of TW Hya are however needed to address this issue.

\section{Summary and conclusions}

Our $200 \mathrm{ks}$ Chandra observation has proven that V2129 Oph is one of the X-ray brightest CTTS, with $L_{\mathrm{X}}=2.7 \times 10^{30} \mathrm{erg} \mathrm{s}^{-1}$ and $\log L_{\mathrm{X}} / L_{\mathrm{bol}}=-3.3$. The hottest components of the X-ray emitting plasma showed flaring activity typical of active coronae. In contrast the plasma at temperature of 3-4 MK has a high density, similar to that observed in almost all CTTS with measured densities, and contrasting with a coronal origin. For the first time, our Chandra observation of V2129 Oph has provided evidence of time variable density: during the first half of the observation the plasma at 3-4 MK had high density $\left(\log N_{\mathrm{e}} \approx 12.1\right.$ ), while during the second half it had lower density, although the statistical significance of this density difference is marginal. The fluxes of the strongest emission lines produced by this cool plasma component, which probe the $E M$, are correlated with plasma density: in the first half of the observation, they were significantly higher than in the second.

These results confirm that the accretion phenomenon is associated with the presence of high density plasma at a few MK, which is thus likely to be material heated in the accretion shock. They also suggest that variations in the observed density and $E M$ of this plasma component can occur over timescales of $\sim 100 \mathrm{ks}$.

Assuming that this high density plasma is material heated in the accretion shock, these observed variations can be interpreted in terms of rotational modulation: in fact, the viewing geometry of the accretion funnel, as determined through simultaneous optical observations, changes significantly during the Chandra observation. During the first half of the observing time the X-rays produced in the accretion shock region are observable, while during the second half of the observation the dense portions of the accretion funnel near the shock blocks the accretion-driven $\mathrm{X}$-rays emitted in the direction of the observer.

Acknowledgements. We thank Fabio Reale for useful suggestions on flare analysis. S.G.G. acknowledges the support of the Science and Technology Facilities Council [grant number ST/G006261/1].

\section{References}

Aarnio, A. N., Stassun, K. G., \& Matt, S. P. 2010, ApJ, 717, 93

Alexander, R. D., Clarke, C. J., \& Pringle, J. E. 2004, MNRAS, 354, 71

Argiroffi, C., Maggio, A., \& Peres, G. 2007, A\&A, 465, L5

Argiroffi, C., Maggio, A., Peres, G., et al. 2009, A\&A, 507, 939

Asplund, M., Grevesse, N., \& Sauval, A. J. 2005, in Cosmic Abundances as Records of Stellar Evolution and Nucleosynthesis, ed. T. G. Barnes III, \& F. N. Bash, ASP Conf. Ser., 336, 25

Baraffe, I., Chabrier, G., \& Gallardo, J. 2009, ApJ, 702, L27

Batalha, C., Batalha, N. M., Alencar, S. H. P., Lopes, D. F., \& Duarte, E. S. 2002, ApJ, 580, 343

Bertout, C. 1989, ARA\&A, 27, 351

Bouvier, J., \& Appenzeller, I. 1992, A\&AS, 92, 481

Bouvier, J., Cabrit, S., Fernandez, M., Martin, E. L., \& Matthews, J. M. 1993, A\&A, 272, 176

Bouvier, J., Alencar, S. H. P., Boutelier, T., et al. 2007, A\&A, 463, 1017

Brickhouse, N. S., Cranmer, S. R., Dupree, A. K., Luna, G. J. M., \& Wolk, S. 2010, ApJ, 710, 1835

Canizares, C. R., Davis, J. E., Dewey, D., et al. 2005, PASP, 117, 1144

Casanova, S., Montmerle, T., Feigelson, E. D., \& Andre, P. 1995, ApJ, 439, 752

Curran, R. L., Argiroffi, C., Sacco, G. G., et al. 2011, A\&A, 526, A104

Donati, J., Jardine, M. M., Gregory, S. G., et al. 2007, MNRAS, 380, 1297

Donati, J., Jardine, M. M., Gregory, S. G., et al. 2009, in EAS PS, ed. C. Neiner, \& J.-P. Zahn, 39, 133

Donati, J., Bouvier, J., Walter, F. M., et al. 2011, MNRAS, 154

Drake, J. J. 2005, in 13th Cambridge Workshop on Cool Stars, Stellar Systems and the Sun, ed. F. Favata, G. A. J. Hussain, \& B. Battrick, ESA SP, 560, 519 Ercolano, B., Clarke, C. J., \& Drake, J. J. 2009, ApJ, 699, 1639

Favata, F., Flaccomio, E., Reale, F., et al. 2005, ApJS, 160, 469

Flaccomio, E., Micela, G., Favata, F., \& Alencar, S. P. H. 2010, A\&A, 516, L8

Gabriel, A. H., \& Jordan, C. 1969, MNRAS, 145, 241

Getman, K. V., Feigelson, E. D., Micela, G., et al. 2008, ApJ, 688, 437

Grankin, K. N., Bouvier, J., Herbst, W., \& Melnikov, S. Y. 2008, A\&A, 479, 827

Gregory, S. G., Wood, K., \& Jardine, M. 2007, MNRAS, 379, L35

Gregory, S. G., Flaccomio, E., Argiroffi, C., et al. 2009, in High Resolution Xray Spectroscopy: Towards IXO

Güdel, M., \& Telleschi, A. 2007, A\&A, 474, L25

Güdel, M., Skinner, S. L., Mel'Nikov, S. Y., et al. 2007, A\&A, 468, 529

Gullbring, E. 1994, A\&A, 287, 131

Günther, H. M., Liefke, C., Schmitt, J. H. M. M., Robrade, J., \& Ness, J. 2006, A\&A, 459, L29

Günther, H. M., Schmitt, J. H. M. M., Robrade, J., \& Liefke, C. 2007, A\&A, 466,1111

Günther, H. M., Matt, S. P., Schmitt, J. H. M. M., et al. 2010, A\&A, 519, A97

Huenemoerder, D. P., Kastner, J. H., Testa, P., Schulz, N. S., \& Weintraub, D. A. 2007, ApJ, 671, 592

Jardine, M. M., Gregory, S. G., \& Donati, J. 2008, MNRAS, 386, 688

Kashyap, V., \& Drake, J. J. 1998, ApJ, 503, 450

Kashyap, V., \& Drake, J. J. 2000, Bull. Astron. Soc. India, 28, 475

Kastner, J. H., Huenemoerder, D. P., Schulz, N. S., Canizares, C. R., \& Weintraub, D. A. 2002, ApJ, 567, 434

Kastner, J. H., Huenemoerder, D. P., Schulz, N. S., et al. 2004, ApJ, 605, L49

Kenyon, S. J., \& Hartmann, L. 1995, ApJS, 101, 117

Königl, A. 1991, ApJ, 370, L39

Maggio, A., Flaccomio, E., Favata, F., et al. 2007, ApJ, 660, 1462

McCabe, C., Ghez, A. M., Prato, L., et al. 2006, ApJ, 636, 932

Orlando, S., Sacco, G. G., Argiroffi, C., et al. 2010, A\&A, 510, A71

Preibisch, T., Kim, Y., Favata, F., et al. 2005, ApJS, 160, 401

Qi, C., Ho, P. T. P., Wilner, D. J., et al. 2004, ApJ, 616, L11

Reale, F. 2007, A\&A, 471, 271

Reale, F., Betta, R., Peres, G., Serio, S., \& McTiernan, J. 1997, A\&A, 325, 782

Robrade, J., \& Schmitt, J. H. M. M. 2007, A\&A, 473, 229

Sacco, G. G., Argiroffi, C., Orlando, S., et al. 2008, A\&A, 491, L17

Sacco, G. G., Orlando, S., Argiroffi, C., et al. 2010, A\&A, 522, A55

Schmitt, J. H. M. M., Robrade, J., Ness, J., Favata, F., \& Stelzer, B. 2005, A\&A, 432, L35

Shevchenko, V. S., \& Herbst, W. 1998, AJ, 116, 1419

Shu, F., Najita, J., Ostriker, E., et al. 1994, ApJ, 429, 781

Siess, L., Dufour, E., \& Forestini, M. 2000, A\&A, 358, 593

Stelzer, B., \& Schmitt, J. H. M. M. 2004, A\&A, 418, 687

Testa, P., Drake, J. J., \& Peres, G. 2004, ApJ, 617, 508

Vuong, M. H., Montmerle, T., Grosso, N., et al. 2003, A\&A, 408, 581

Young, P. R., Del Zanna, G., Landi, E., et al. 2003, ApJS, 144, 135 